Supporting Information for

\title{
Alkyl-Substituted Selenium-Bridged V-Shaped Organic Semiconductors Exhibiting High Hole Mobility and Unusual Aggregation Behavior
}

Toshihiro Okamoto*, ${ }^{1,2,3}$, Masato Mitani ${ }^{1}$, Craig P. Yu ${ }^{1}$, Chikahiko Mitsui ${ }^{1}$, Masakazu Yamagishi $^{4}$, Hiroyuki Ishii ${ }^{5}$, Go Watanabe $^{6}$, Shohei Kumagai ${ }^{1}$, Daisuke Hashizume ${ }^{7}$, Shota Tanaka ${ }^{8}$, Masafumi Yano $^{8}$, Tomokatsu Kushida ${ }^{1}$, Hiroyasu Sato ${ }^{9}$, Kunihisa Sugimoto $^{10,11}$, Takashi Kato ${ }^{12}$, and Jun Takeya ${ }^{1,2,13}$

${ }^{1}$ Material Innovation Research Center (MIRC) and Department of Advanced Materials Science, School of Frontier Sciences, The University of Tokyo, 5-1-5 Kashiwanoha, Kashiwa, Chiba 277-8561, Japan. ${ }^{2}$ National Institute of Advanced Industrial Science and Technology (AIST)-University of Tokyo Advanced Operando-Measurement Technology Open Innovation Laboratory (OPERANDO-OIL), AIST, 5-1-5 Kashiwanoha, Kashiwa, Chiba 277-8561, Japan. ${ }^{3}$ PRESTO, JST, 4-1-8 Honcho, Kawaguchi, Saitama 332-0012, Japan. ${ }^{4}$ National Institute of Technology, Toyama College, 13 Hongo-machi, Toyama, Toyama 939-8630, Japan. ${ }^{5}$ Department of Applied Physics, Faculty of Pure and Applied Sciences, University of Tsukuba, 1-1-1 Tennodai, Tsukuba, Ibaraki 305-8573, Japan. ${ }^{6}$ Department of Physics, School of Science, Kitasato University, 1-15-1 Kitasato, Minami-ku, Sagamihara, Kanagawa 252-0373, Japan. ${ }^{7}$ RIKEN Center for Emergent Matter Science (CEMS), 2-1 Hirosawa, Wako, Saitama 351-0198, Japan. ${ }^{8}$ Chemistry, Materials and Bioengineering Major, Graduate School of Science and Engineering, Kansai University, 3-3-35 Yamate-cho, Suita, Osaka 564-8680, Japan. ${ }^{9}$ Rigaku Corp., 3-9-12 Matsubara-cho, Akishima, Tokyo 196-8666, Japan. ${ }^{10}$ Diffraction \& Scattering Division, Japan Synchrotron Radiation Research Institute, 1-1-1 Kouto, Sayo-cho, Sayo-gun, Hyogo 679-5198, Japan. ${ }^{11}$ Institute for Integrated Cell-Material Sciences (iCeMS), Kyoto University, Yoshida-Honmachi, Sakyo-ku, Kyoto 606-8501, Japan. ${ }^{12}$ Department of Chemistry and Biotechnology, School of Engineering, The University of Tokyo, 7-3-1 Hongo, Bunkyo-ku, Tokyo 113-8656, Japan. ${ }^{13}$ International Center for Materials Nanoarchitectonics (MANA), National Institute for Materials Science (NIMS), 1-1 Namiki, Tsukuba 205-0044, Japan. 


\section{Contents}

1. Synthesis and Characterization

2. Thermal Analyses: Thermogravimetric Analyses (TGA)

3. Ionization Potentials

4. Single-Crystal Analyses

5. Differential Scanning Calorimetric (DSC) Analyses

6. Powder X-ray Analyses

7. Powder UV-vis Absorption Spectra

8. Time-Dependent Absorption Spectra in Solution and Deposited Thin Film

9. Theoretical Calculations

10. X-ray Diffraction Measurements and AFM Observation for Polycrystalline Films

11. OFET Device Fabrication and Evaluation Procedure

12. References 


\section{Materials and General for Synthesis and Characterization}

Materials

Reagents and Starting Materials: 2,7-Dibromonaphthalene, trimethylsilyl chloride, 2,2,6,6-tetramethylpiperidine (TMP), isopropylmagnesium bromide (i-PrMgBr), and tetrakis(triphenylphosphine)palladium(0) were purchased from TCI. TMP was distilled prior to use. Zinc chloride (99.999\% trace metals basis), selenium (99.99\% trace metals basis), decyl magnesium bromide in diethyl ether, and iodine monochloride (ICl) in dichloromethane were purchased from Sigma-Aldrich Inc. 1-Methyl-2-pyrrolidone (NMP) and sulfuryl chloride $\left(\mathrm{SO}_{2} \mathrm{Cl}_{2}\right)$ were purchased from Wako chemicals. Lithium chloride, $n$-BuLi in hexane, $t$-BuLi in pentane, and iodine, and all anhydrous solvents were purchased from KANTO chemicals.

\section{General for Synthesis and Characterization}

All reactions were carried out under an atmosphere of either nitrogen or argon. Air- and moisture-sensitive liquids and solutions were transferred via a syringe or a Teflon cannula. Analytical thin-layer chromatography (TLC) was performed on glass plates with $0.25 \mathrm{~mm} 230-400$ mesh silica gel containing a fluorescent indicator (Merck Silica gel 60 F254). TLC plates were visualized by either exposure to ultraviolet lamp (254 nm and $365 \mathrm{~nm}$ ) or by dipping with $10 \%$ phosphomolybdic acid in ethanol and then heating on a hot plate. Flash column chromatography was performed on Kanto silica gel 60. Open column chromatography was performed on Wakogel C-200 $(75-150 \mu \mathrm{m})$. All NMR spectra were recorded on a JEOL ECA600 and JEOL ECS400 spectrometers. Chemical shifts are reported in parts per million ( $\mathrm{ppm}, \delta$ scale) from residual protons in the

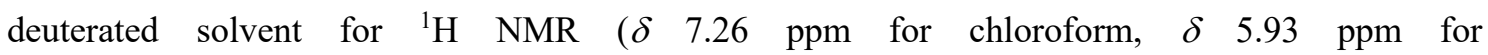
1,1,2,2-tetrachloroethane) and from the solvent carbon for ${ }^{13} \mathrm{C}$ NMR $(\delta 77.16 \mathrm{ppm}$ for chloroform and $\delta 73.78 \mathrm{ppm}$ for 1,1,2,2-tetrachloroethane). The data were presented in the following format: chemical shift, multiplicity ( $\mathrm{s}=$ singlet, $\mathrm{d}=$ doublet, $\mathrm{t}=$ triplet, $\mathrm{m}=$ multiplet), coupling constant in Hertz $(\mathrm{Hz})$, signal area integration in natural numbers, assignment (italic). Mass spectra were measured on a JEOL JMS-T100LC APCI/ESI mass spectrometer. Melting points and elemental analyses were collected on a Mettler Toledo MP70 Melting Point System and J-Science Lab JM10 MICRO CORDER, respectively.

\section{Zinc chloride solution}

An oven-dried Schlenk tube was charged with zinc chloride (52.3 g, $384 \mathrm{mmol})$, and the tube was heated under evacuation until zinc chloride completely melted. After cooling to room temperature, dry THF $(384 \mathrm{~mL})$ was added to give ca. $1.0 \mathrm{M}$ zinc chloride solution. 


\section{LiTMP}

To a solution of TMP $(65.3 \mathrm{~mL}, 384 \mathrm{mmol})$ in THF $(384 \mathrm{~mL})$ was added $n$-BuLi $(1.60 \mathrm{M}$ hexane solution, $240 \mathrm{~mL}, 384 \mathrm{mmol}$ ) at $-78{ }^{\circ} \mathrm{C}$. After stirred at $0{ }^{\circ} \mathrm{C}$ for $30 \mathrm{~min}$, LiTMP solution was generated.

\section{$t-\mathrm{Bu}_{2} \mathrm{Zn}(\mathrm{TMP}) \mathrm{Li}^{1-3}$}

To a solution of $\mathrm{ZnCl}_{2}(1.0 \mathrm{M}$ in THF, $274 \mathrm{~mL}, 274 \mathrm{mmol})$ was added $t$-BuLi (1.71 M pentane solution, $434 \mathrm{~mL}, 768 \mathrm{mmol}$ ) at $0{ }^{\circ} \mathrm{C}$, and the mixture was stirred at $0{ }^{\circ} \mathrm{C}$ for $30 \mathrm{~min}$. The prepared $t$ - $\mathrm{Bu}_{2} \mathrm{Zn}$ was transferred via a Teflon cannula to a solution of LiTMP at $-78{ }^{\circ} \mathrm{C}$. After stirred at $0{ }^{\circ} \mathrm{C}$ for $30 \mathrm{~min}, t-\mathrm{Bu}_{2} \mathrm{Zn}(\mathrm{TMP}) \mathrm{Li}$ solution was prepared.

\section{$\mathrm{SeCl}_{2}{ }^{4}$}

To selenium powder (474 mg, $6.0 \mathrm{mmol}, 1.20 \mathrm{~mol}$ amt.) was added $\mathrm{SO}_{2} \mathrm{Cl}_{2}(810 \mathrm{mg}, 6.0 \mathrm{mmol}, 1.20$ mol amt.) at ambient temperature. The mixture was stirred for $10 \mathrm{~min}$, and $12 \mathrm{~mL}$ of THF was added. The solution was stirred for $1 \mathrm{~h}$ to give a clear brownish red solution of $\mathrm{SeCl}_{2}$.

\section{7-Bromo-2-trimethylsilylnaphthalene}

To a suspension of 2,7-dibromonaphthalene $(50.0 \quad \mathrm{~g}, \quad 175 \mathrm{mmol})$ and $N, N, N^{\prime}, N^{\prime}$-tetramethylethylenediamine (28.7 mL, $193 \mathrm{mmol}, 1.10 \mathrm{~mol}$ amt.) in $\mathrm{Et}_{2} \mathrm{O}(350 \mathrm{~mL})$ was added $n$-BuLi (1.60 M in hexane, $113 \mathrm{~mL}, 180 \mathrm{mmol}, 1.03 \mathrm{~mol}$ amt.) dropwise over $20 \mathrm{~min}$ at -78 ${ }^{\circ} \mathrm{C}$. After the mixture was stirred at that temperature for $1.5 \mathrm{~h}$, a solution of trimethylsilyl chloride (26.7 mL, $210 \mathrm{mmol}, 1.20 \mathrm{~mol} \mathrm{amt}$.) in $\mathrm{Et}_{2} \mathrm{O}(210 \mathrm{~mL})$ was added at $-78{ }^{\circ} \mathrm{C}$. After stirring at that temperature for $3 \mathrm{~h}$, the reaction was quenched by $\mathrm{H}_{2} \mathrm{O}$. The organic layer was separated with hexane, washed with brine, dried over $\mathrm{MgSO}_{4}$. After removing the solvent was removed in vacuo, the crude material was purified by distillation $\left(151{ }^{\circ} \mathrm{C}, 2.9\right.$ Torr) to afford the titled compound (39.6 g, 142 mmol, 81\%) as white solid. m.p.: 41.7-42.2 ${ }^{\circ} \mathrm{C} .{ }^{1} \mathrm{H}$ NMR $\left(400 \mathrm{MHz}, \mathrm{CDCl}_{3}\right): \delta 0.34$ (s, 9H, $\left.\mathrm{Si}\left(\mathrm{CH}_{3}\right)_{3}\right), 7.54(\mathrm{dd}, J=8.0 \mathrm{~Hz}$ and $1.6 \mathrm{~Hz}, 1 \mathrm{H}, \mathrm{Ar} H), 7.60(\mathrm{~d}, J=8.0 \mathrm{~Hz}, 1 \mathrm{H}, \mathrm{Ar} H), 7.68(\mathrm{~d}, J=8.0$ $\mathrm{Hz}, 1 \mathrm{H}, \operatorname{Ar} H), 7.78(\mathrm{~d}, J=8.0 \mathrm{~Hz}, 1 \mathrm{H}, \operatorname{Ar} H), 7.90(\mathrm{~s}, 1 \mathrm{H}, \operatorname{Ar} H), 8.01(\mathrm{~d}, J=1.6 \mathrm{~Hz}, 1 \mathrm{H}, \operatorname{Ar} H) .{ }^{13} \mathrm{C}$ NMR (100 MHz, $\left.\mathrm{CDCl}_{3}\right): \delta-1.05,119.77,126.90,129.47,129.62,130.16,130.35,132.05,132.84$, 134.03, 139.54. TOF HRMS (APCI+): Calcd for $\mathrm{C}_{13} \mathrm{H}_{16}{ }^{79} \mathrm{BrSi}[\mathrm{M}+\mathrm{H}]$ 278.0121, found, 278.0117. Anal. Calcd for $\mathrm{C}_{13} \mathrm{H}_{15} \mathrm{BrSi}$ : C, 55.91; H, 5.41. Found C, 56.19; H, 5.68.

\section{2-Bromo-3-iodo-7-trimethylsilylnaphthalene (2)}

To a suspension of $t-\mathrm{Bu}_{2} \mathrm{Zn}(\mathrm{TMP}) \mathrm{Li}$ ( $384 \mathrm{mmol}, 2.4 \mathrm{~mol} \mathrm{amt}$.) was added a solution of compound 1 $(44.7 \mathrm{~g}, 160 \mathrm{mmol})$ in THF $(240 \mathrm{~mL})$ dropwise at $-78{ }^{\circ} \mathrm{C}$. After stirring at $-20{ }^{\circ} \mathrm{C}$ for $12 \mathrm{~h}$, a solution of iodine $(284 \mathrm{~g}, 1.12 \mathrm{~mol}, 7.0 \mathrm{~mol} \mathrm{amt}$ ) $)$ in THF $(373 \mathrm{~mL})$ was added at $-78^{\circ} \mathrm{C}$. After 
stirring at this temperature for $2 \mathrm{~h}$, the reaction was quenched by $\mathrm{Na}_{2} \mathrm{~S}_{2} \mathrm{O}_{3}$ aqueous solution. The organic phase was separated with ethyl acetate, washed with brine, and dried over $\mathrm{MgSO}_{4}$. After removing the solvent in vacuo, the crude material was passed through a short pad of silica gel column employing hexane as an eluent. After removing the solvent in vacuo, the crude materials were purified by recrystallization from hexane/chloroform to afford the titled compound 2 ( $40.2 \mathrm{~g}$, $99.2 \mathrm{mmol}, 62 \%)$ as white solid. m.p.: $79.2-80.0{ }^{\circ} \mathrm{C} .{ }^{1} \mathrm{H}$ NMR $\left(400 \mathrm{MHz}, \mathrm{CDCl}_{3}\right): \delta 0.33(\mathrm{~s}, 9 \mathrm{H}$, $\left.\mathrm{Si}\left(\mathrm{CH}_{3}\right)_{3}\right), 7.61(\mathrm{~d}, J=8.0 \mathrm{~Hz}, 1 \mathrm{H}, \operatorname{Ar} H), 7.67$ (d, $\left.J=8.0 \mathrm{~Hz}, 1 \mathrm{H}, \operatorname{Ar} H\right), 7.86(\mathrm{~s}, 1 \mathrm{H}, \operatorname{Ar} H), 8.15$ (s, $1 \mathrm{H}, \operatorname{Ar} H), 8.39$ (s, $1 \mathrm{H}, \operatorname{Ar} H) .{ }^{13} \mathrm{C}$ NMR $\left(100 \mathrm{MHz}, \mathrm{CDCl}_{3}\right): \delta-1.12,98.58,125.62,126.01,131.04$, 131.17, 132.65, 133.26, 133.46, 139.47, 140.32. TOF HRMS (APCI+): Calcd for $\mathrm{C}_{13} \mathrm{H}_{15}{ }^{79} \mathrm{BrSi}$ $[\mathrm{M}+\mathrm{H}]$ 403.9087, found, 403.9070. Anal. Calcd for $\mathrm{C}_{13} \mathrm{H}_{14} \mathrm{BrSi}$ : C, 38.54; H, 3.48. Found C, 38.40; $\mathrm{H}, 3.73$.

\section{3,3'-Dibromo-6,6'-bis(trimethylsilyl)-2,2'-binaphthalene (3)}

To a suspension of compound $2(6.89 \mathrm{~g}, 17.0 \mathrm{mmol})$ in THF $(68 \mathrm{~mL}, 0.25 \mathrm{M})$ was added $i-\mathrm{PrMgBr}$ (1.0 M in THF, $17.9 \mathrm{~mL}, 17.9 \mathrm{mmol}, 1.03 \mathrm{~mol}$ amt.) at $-78{ }^{\circ} \mathrm{C}$. After stirring at that temperature for $30 \mathrm{~min}$, a solution of $\mathrm{ZnCl}_{2}(1.0 \mathrm{M}$ in THF, $17.9 \mathrm{~mL}, 17.9 \mathrm{mmol}, 1.03 \mathrm{~mol}$ amt.) was added at that temperature. After warming to ambient temperature and stirring for $30 \mathrm{~min}, \mathrm{LiCl}(0.5 \mathrm{M}$ in THF, $35.8 \mathrm{~mL}, 35.8 \mathrm{mmol}, 1.03 \mathrm{~mol} \mathrm{amt}$.) was added and stirred for $30 \mathrm{~min}$ at ambient temperature. To the resulting colorless solution, $N$-methyl-2-pyrrolidone $(17 \quad \mathrm{~mL})$, 2-bromo-3-iodo-7-trimethylsilylnaphthalene $(6.54 \mathrm{~g}, 16.2 \mathrm{mmol}, \quad 0.95 \mathrm{~mol}$ amt. $)$ and tetrakis(triphenylphosphine)palladium(0) (374 $\mathrm{mg}, 0.320 \mathrm{mmol}, 2 \mathrm{~mol} \%)$ was added. After the mixture was stirred at ambient temperature for $16 \mathrm{~h}$, the reaction was quenched by $\mathrm{MeOH}$. The resulting white precipitate was collected by filtration. After dissolving the collected white solid in chloroform, the organic materials were passed through a short pad of silica gel column. After reducing the solvent to c.a. $10 \mathrm{~mL}$ in vacuo, addition of $\mathrm{MeOH}$ afforded the titled compound 3 (7.21 g, $13.0 \mathrm{mmol}, 80 \%$ yield) as white solid, which was collected by filtration. After removing the solvent of the combined filtrate in vacuo, the crude material was purified by silica gel column chromatography (hexane: chloroform $=98: 2$ to $95: 5$ ) to afford the additional titled compound 3 (1.35 g, 2.4 mmol, 15\% yield). Total yield: 95\%. m.p.: 256.2-256.9 ${ }^{\circ} \mathrm{C} .{ }^{1} \mathrm{H}$ NMR (400 MHz, $\left.\mathrm{CDCl}_{3}\right): \delta 0.37\left(\mathrm{~s}, 18 \mathrm{H}, \mathrm{Si}\left(\mathrm{CH}_{3}\right)_{3}\right), 7.66(\mathrm{~d}, J=8.0 \mathrm{~Hz}, 2 \mathrm{H}, \mathrm{Ar} H), 7.77(\mathrm{~s}, 2 \mathrm{H}, \mathrm{Ar} H), 7.81(\mathrm{~d}, J=8.0$ $\mathrm{Hz}, 2 \mathrm{H}, \mathrm{Ar} H$ ), 7.99 (s, $2 \mathrm{H}, \mathrm{Ar} H$ ), 8.21 (s, $2 \mathrm{H}, \mathrm{Ar} H) .{ }^{13} \mathrm{C} \mathrm{NMR}\left(100 \mathrm{MHz}, \mathrm{CDCl}_{3}\right): \delta-1.00,121.87$, $127.05,129.98,130.81,131.24,132.22,132.70,133.52,139.96,140.01$. TOF HRMS (APCI+): Calcd for $\mathrm{C}_{26} \mathrm{H}_{29}{ }^{79} \mathrm{Br}_{2} \mathrm{Si}_{2}[\mathrm{M}+\mathrm{H}]$ 554.0091, found, 554.0076. Anal. Calcd for $\mathrm{C}_{26} \mathrm{H}_{28} \mathrm{Br}_{2} \mathrm{~S}_{2}$ : C, 56.12; H, 5.07. Found C, 56.12; H, 5.18. 


\section{3,9-Bis(trimethylsilyl)dinaphtho[2,3-b:2',3'-d] selenophene (4)}

To a white suspension of compound $3(2.78 \mathrm{~g}, 5.00 \mathrm{mmol})$ in $\mathrm{Et}_{2} \mathrm{O}(25 \mathrm{~mL})$ was added $n$-BuLi in hexane $\left(1.60 \mathrm{M}, 6.44 \mathrm{~mL}, 10.3 \mathrm{mmol}, 2.05 \mathrm{~mol}\right.$ amt.) at $-78{ }^{\circ} \mathrm{C}$. After warming to $0{ }^{\circ} \mathrm{C}$ and stirring for $1 \mathrm{~h}$, the prepared $\mathrm{SeCl}_{2}$ was transferred by Teflon cannula to the resulting brown solution at $-78^{\circ} \mathrm{C}$. After stirring at $-78^{\circ} \mathrm{C}$ for $2 \mathrm{~h}$, the reaction was quenched with $\mathrm{H}_{2} \mathrm{O}$. The organic layer was extracted with $\mathrm{CH}_{2} \mathrm{Cl}_{2}$, washed with brine, dried over $\mathrm{MgSO}_{4}$. After removing the solvent in vacuo, the crude material was purified by silica gel column chromatography (hexane: dichloromethane $=$ 100:0 to 95:5) to afford the title compound 4 (1.52 g, 3.20 mmol, 64\% yield). m.p.: $271.0-271.9^{\circ} \mathrm{C}$. ${ }^{1} \mathrm{H}$ NMR (400 MHz, $\left.\mathrm{CDCl}_{3}\right): \delta 0.38\left(\mathrm{~s}, 18 \mathrm{H}, \mathrm{Si}\left(\mathrm{CH}_{3}\right)_{3}\right), 7.64$ (d, $\left.J=8.0 \mathrm{~Hz}, 2 \mathrm{H}, \mathrm{Ar} H\right), 8.01(\mathrm{~d}, J=$ $8.0 \mathrm{~Hz}, 2 \mathrm{H}, \operatorname{Ar} H) 8.02$ (s, 2H, $\operatorname{Ar} H$ ), 8.27 (s, 2H, ArH), 8.68 (s, 2H, ArH). ${ }^{13} \mathrm{C}$ NMR (100 MHz, $\left.\mathrm{CDCl}_{2} \mathrm{CDCl}_{2}\right): \delta-0.84,121.66,124.61,127.62,129.81,131.42,132.72,132.87,136.46,137.52$, 139.31. HRMS (APCI+): Calcd for $\mathrm{C}_{26} \mathrm{H}_{29} \mathrm{SeSi}_{2}[\mathrm{M}+\mathrm{H}]$ 477.0973, found, 477.0974. Anal. Calcd for $\mathrm{C}_{26} \mathrm{H}_{28} \mathrm{SeSi}_{2}$ : C, 65.66; H, 5.93. Found C, 65.35; H, 5.85.

\section{3,9-Diiododinaphtho[2,3-b:2',3'-d] selenophene (I-DNS-VW)}

To a pale yellow suspension of $4(686 \mathrm{mg}, 1.60 \mathrm{mmol})$ in dichloromethane $(16 \mathrm{~mL})$ was added ICl in dichloromethane $(1.0 \mathrm{M}, 3.68 \mathrm{~mL}, 3.68 \mathrm{mmol}, 2.3 \mathrm{~mol}$ amt. $)$ was at $-78{ }^{\circ} \mathrm{C}$. After stirring at $0{ }^{\circ} \mathrm{C}$ for $2 \mathrm{~h}$, the reaction was quenched by $\mathrm{Na}_{2} \mathrm{~S}_{2} \mathrm{O}_{3}$ aqueous solution and warmed to room temperature. The yellow precipitate was collected by filtration. After dissolving the organic material in 1,2-dichlorobenzene at $120^{\circ} \mathrm{C}$, the solution was passed through a short pad of Celite to remove the insoluble materials. After removing the solvent in vacuo, the title compound I-DNS-VW (783 mg, $1.46 \mathrm{mmol}, 91 \%$ yield) was obtained as yellow solid. m.p.: $343.7-344.5^{\circ} \mathrm{C} .{ }^{1} \mathrm{H}$ NMR $(400 \mathrm{MHz}$, $\mathrm{CDCl}_{2} \mathrm{CDCl}_{2}$ ): $\delta 7.72(\mathrm{~s}, 4 \mathrm{H}, \operatorname{Ar} H), 8.10(\mathrm{~s}, 2 \mathrm{H}, \mathrm{Ar} H), 8.21$ (s, $\left.2 \mathrm{H}, \operatorname{Ar} H\right), 8.60(\mathrm{~s}, 2 \mathrm{H}, \operatorname{Ar} H) .{ }^{13} \mathrm{C}$ NMR (100 MHz, $\left.\mathrm{CDCl}_{2} \mathrm{CDCl}_{2}, 125^{\circ} \mathrm{C}\right): \delta 92.46,121.75,123.16,129.93,130.04,134.33,134.79$, 135.57, 137.51, 137.62. TOF HRMS (APCI+): Calcd for $\mathrm{C}_{20} \mathrm{H}_{11} \mathrm{SeI}_{2}[\mathrm{M}+\mathrm{H}] 584.8115$, found, 584.8114. Anal. Calcd for $\mathrm{C}_{20} \mathrm{H}_{10} \mathrm{SeI}_{2}$ : C, 41.20; H, 1.73. Found C, 41.11; H, 1.86.

\section{3,9-Didecyldinaphtho[2,3-b:2',3'-d] selenophene ( $\mathrm{C}_{10}$-DNS-VW)}

To a colorless solution of decylmagnesium bromide in diethylether (1.0 M, $720 \mu \mathrm{L}, 0.720 \mathrm{mmol}, 2.4$ mol amt.) in toluene (3.0 mL) was added $\mathrm{ZnCl}_{2}$ in THF (1.0 M, $750 \mu \mathrm{L}, 0.750 \mathrm{mmol}, 2.5 \mathrm{~mol}$ amt.) at $0{ }^{\circ} \mathrm{C}$ to prepare organozinc reagent. After stirring at room temperature for $10 \mathrm{~min}$, I-DNS-VW (175 mg, $0.300 \mathrm{mmol}, 1.00 \mathrm{~mol} \mathrm{amt}$.) and $\mathrm{PdCl}_{2}(\mathrm{dppf}) \cdot \mathrm{CH}_{2} \mathrm{Cl}_{2}(9.8 \mathrm{mg}, 0.012 \mathrm{mmol}, 4 \mathrm{~mol} \%)$ were successively added at room temperature. The resulting brown suspension was warmed to $70{ }^{\circ} \mathrm{C}$ and stirred for $12 \mathrm{~h}$. After cooling to room temperature, the reaction was quenched with $\mathrm{H}_{2} \mathrm{O}$. The organic layer was separated by dichloromethane and washed with brine, and dried over $\mathrm{MgSO}_{4}$. After removing the solvent in vacuo, the crude material was purified by silica gel column 
chromatography (hexane: $\mathrm{CH}_{2} \mathrm{Cl}_{2}=100: 0$ to $95: 5$ ) to afford the titled compound $\mathbf{C}_{\mathbf{1 0}}-\mathbf{D N S}-\mathbf{V W}$ as a white solid (172 mg, $0.281 \mathrm{mmol}$, 93\% yield). m.p.: 163.9-164.8 ${ }^{\circ} \mathrm{C} .{ }^{1} \mathrm{H}$ NMR (400 MHz, $\left.\mathrm{CDCl}_{2} \mathrm{CDCl}_{2}\right): \delta 0.82\left(\mathrm{t}, J=6.4 \mathrm{~Hz}, 6 \mathrm{H}, \mathrm{CH}_{3}\right), 1.18-1.36\left(\mathrm{~m}, 28 \mathrm{H},\left(\mathrm{CH}_{2}\right)_{7}\right), 1.67$ (q, $J=7.2 \mathrm{~Hz}, 4 \mathrm{H}$, $\left.\mathrm{ArCH}_{2} \mathrm{CH}_{2}\right), 2.75$ (t, $\left.J=7.2 \mathrm{~Hz}, 4 \mathrm{H}, \mathrm{ArCH}_{2}\right), 7.35$ (d, $\left.J=8.4 \mathrm{~Hz}, 2 \mathrm{H}, \mathrm{ArH}\right), 7.57$ (s, 2H, ArH), 7.90 (d, $J=8.4 \mathrm{~Hz}, 2 \mathrm{H}, \mathrm{Ar} H), 8.14$ (s, $2 \mathrm{H}, \mathrm{Ar} H$ ), 8.57 (s, $2 \mathrm{H}, \operatorname{Ar} H) .{ }^{13} \mathrm{C} \mathrm{NMR}\left(100 \mathrm{MHz}, \mathrm{CDCl}_{2} \mathrm{CDCl}_{2}\right.$ ): $\delta$ 14.37, 22.85, 29.46, 29.51, 29.68, 29.74, 29.77, 31.39, 32.02, 36.32, 121.19, 123.73, 125.10, 127.52, 128.37, 129.77, 133.52, 136.31, 136.57, 141.50. HRMS (APCI+): Calcd for $\mathrm{C}_{40} \mathrm{H}_{53} \mathrm{Se}$ $[\mathrm{M}+\mathrm{H}]$ 613.3312, found, 613.3293. Anal. Calcd for $\mathrm{C}_{40} \mathrm{H}_{52} \mathrm{Se}$ : C, 78.53; H, 8.57. Found C, 78.56; $\mathrm{H}$, 8.76 .

\section{Dinaphtho[2,3-b:2',3'-d] selenophene (DNS-V)}

The target compound was prepared according to a modified reported procedure. 1 To a flame-dried Schlenk tube was added I-DNS-VW (30.0 mg, 0.0514 mmol, 1.0 equiv.) and anhydrous THF (1 $\mathrm{mL}, 0.05 \mathrm{M}$ ), and the light-yellow mixture was purged by argon for $5 \mathrm{~min}$. Subsequently, $\mathrm{PdCl}_{2}$ (dppf) (1.88 mg, $0.00257 \mathrm{mmol}, 5 \mathrm{~mol} \%$ ), TMEDA (13.1 mg, $0.113 \mathrm{mmol}, 2.2$ equiv.), and $\mathrm{NaBH}_{4}$ (4.86 mg, $0.129 \mathrm{mmol}, 2.5$ equiv.) were sequentially added to the system. The reaction mixture was stirred at room temperature under argon for $5 \mathrm{~h}$ before it was quenched by water. The compound was extracted by EtOAc and dried over $\mathrm{Na}_{2} \mathrm{SO}_{4}$. The crude was purified by column chromatography using hexanes/ $\mathrm{CHCl}_{3}$ as eluents $(3: 1 \mathrm{v} / \mathrm{v})$ to afford the target compound as a light-yellow solid (15.0 mg, $0.01452 \mathrm{mmol}, 88 \%$ yield). ${ }^{1} \mathrm{H}$ NMR (400 $\mathrm{MHz}, \mathrm{CDCl}_{2} \mathrm{CDCl}_{2}$ ): $\delta 8.66$ (s, 2H, $\operatorname{Ar} H), 8.24(\mathrm{~s}, 2 \mathrm{H}, \operatorname{Ar} H), 8.03-8.01(\mathrm{t}, J=5.6 \mathrm{~Hz}, 3.2 \mathrm{~Hz}, \quad 2 \mathrm{H}, \operatorname{Ar} H), 7.86-7.83(\mathrm{~s}, J=3.6$ $\mathrm{Hz}, 5.6 \mathrm{~Hz}, 2 \mathrm{H}, \operatorname{Ar} H), 7.53-7.50(\mathrm{t}, J=4.0 \mathrm{~Hz}, 4.4 \mathrm{~Hz}, 4 \mathrm{H}, \operatorname{Ar} H) .{ }^{13} \mathrm{C}$ NMR $(100 \mathrm{MHz}$, $\left.\mathrm{CDCl}_{2} \mathrm{CDCl}_{2}, 100{ }^{\circ} \mathrm{C}\right): \delta 137.13,136.28,133.37,131.26,128.31,126.54,126.31,125.39,124.05$, 121.35. TOF HRMS (APCI+): Calcd for $\mathrm{C}_{20} \mathrm{H}_{12} \mathrm{Se}[\mathrm{M}+\mathrm{H}] 333.0182$, found, 333.0177 . 


\section{Thermal Analyses: Thermogravimetric Analyses (TGA)}

TGA measurements were carried out with a Rigaku Thermo Plus EVO II TG 8120. Sample was placed in an aluminum pan and heated at the rate of $5 \mathrm{~K} \mathrm{~min}^{-1}$ under $\mathrm{N}_{2}$ purge at a flow rate of 100 $\mathrm{mL} \min ^{-1} . \mathrm{Al}_{2} \mathrm{O}_{3}$ was used as the reference material. The TG data of DNS-V and $\mathbf{C}_{\mathbf{1 0}}-\mathbf{D N S}-\mathbf{V W}$ along with DNF-V, DNT-V, $\mathbf{C}_{\mathbf{1 0}}-\mathbf{D N F}-\mathbf{V W}$, and $\mathbf{C}_{\mathbf{1 0}}-\mathbf{D N T}-\mathbf{V W}$ are depicted in Figure S1 and S2, respectively.

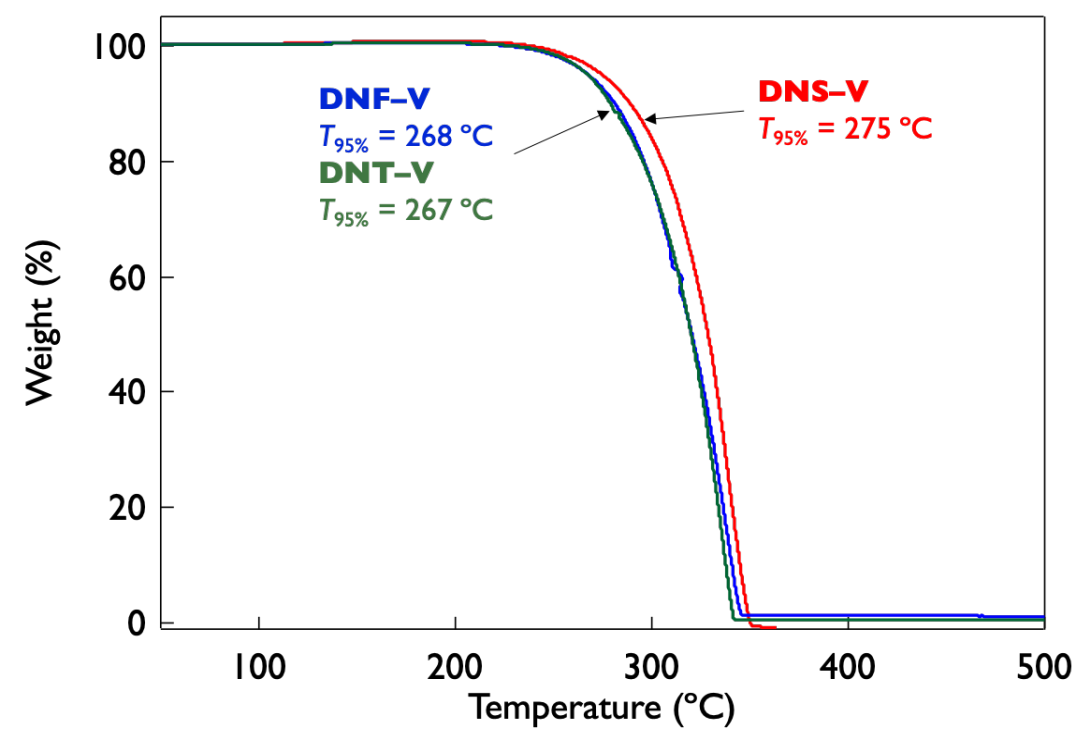

Figure S1. TGA charts of DNF-V, DNT-V and DNS-V.

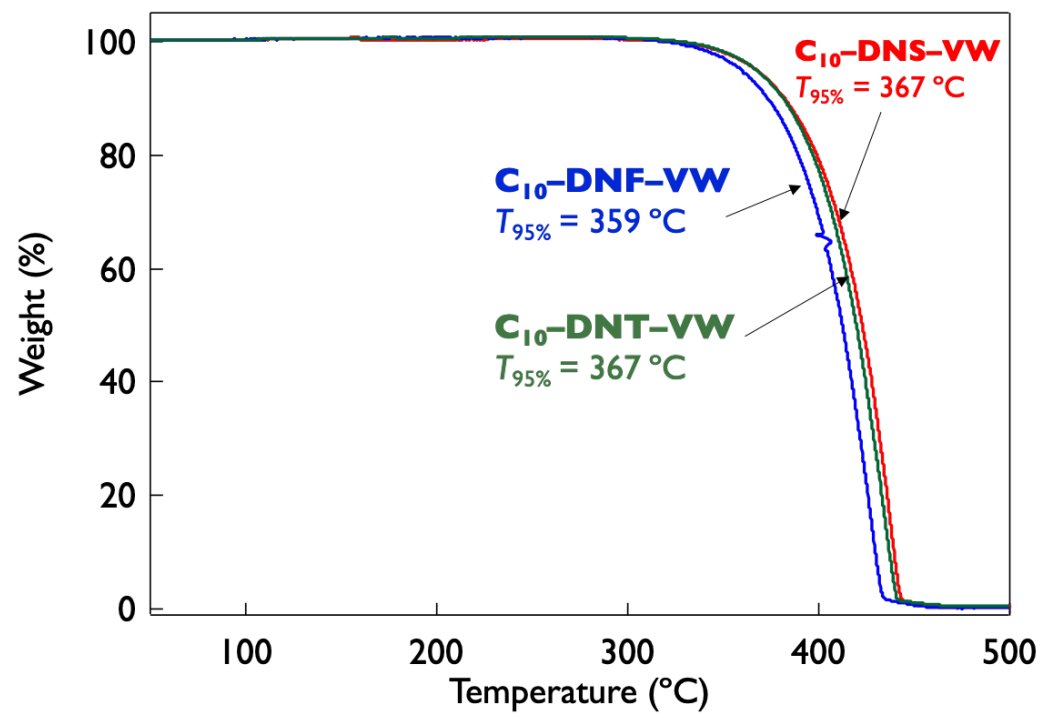

Figure S2. TGA charts of $\mathbf{C}_{10}-\mathbf{D N F}-\mathbf{V W}, \mathbf{C}_{10}-\mathbf{D N T}-\mathbf{V W}$ and $\mathbf{C}_{10}-\mathrm{DNS}-\mathrm{VW}$. 


\section{Ionization Potentials}

Photoelectron yield spectroscopy (PYS) was performed on a Sumitomo Heavy Industries Advanced Machinery PYS-202. For PYS measurements, thin films $(c a .100 \mathrm{~nm})$ of unsubstituted and decyl-substituted V-shaped derivatives were thermally evaporated on ITO coated quartz substrates and measurements were performed in vacuum (Figure S3-4). Table 1 summarized their ionization potentials and calculated HOMO level of DNS-V and $\mathbf{C}_{\mathbf{1 0}}-\mathbf{D N S}-\mathbf{V W}$ along with DNF-V, DNT-V, $\mathrm{C}_{10}-\mathrm{DNF}-\mathrm{VW}$, and $\mathrm{C}_{10}-\mathrm{DNT}-\mathrm{VW}$.

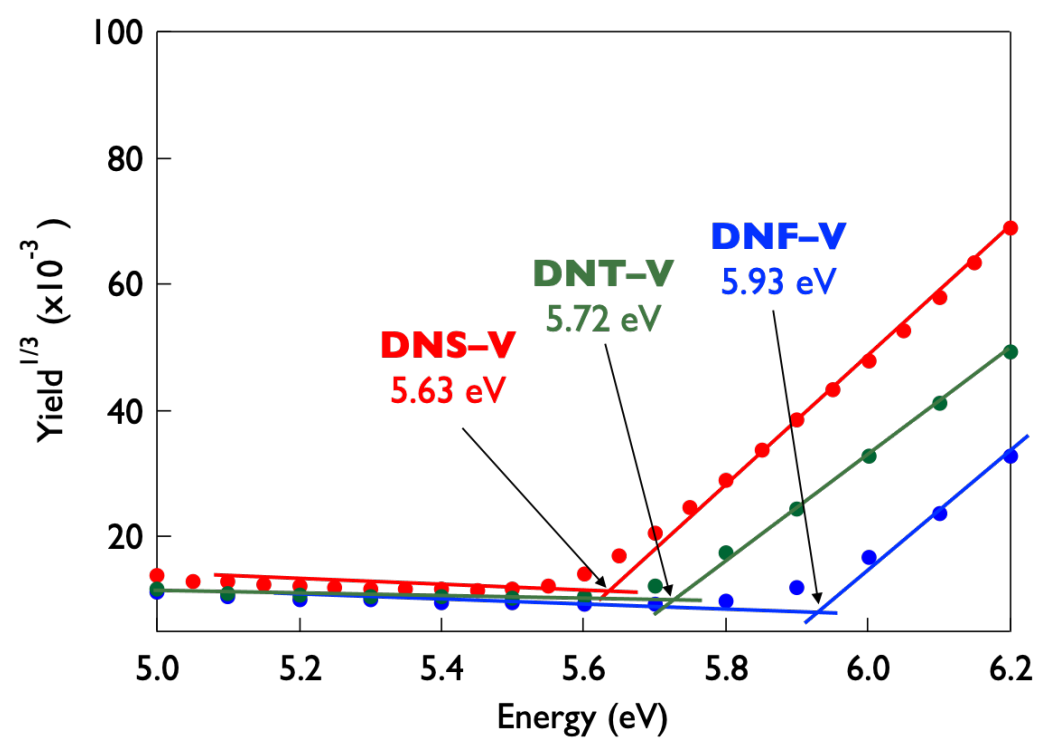

Figure S3. Photoelectron yield spectra of DNF-V, DNT-V and DNS-V in thin films after light illumination from a $\mathrm{D}_{2}$ lamp in vacuo.

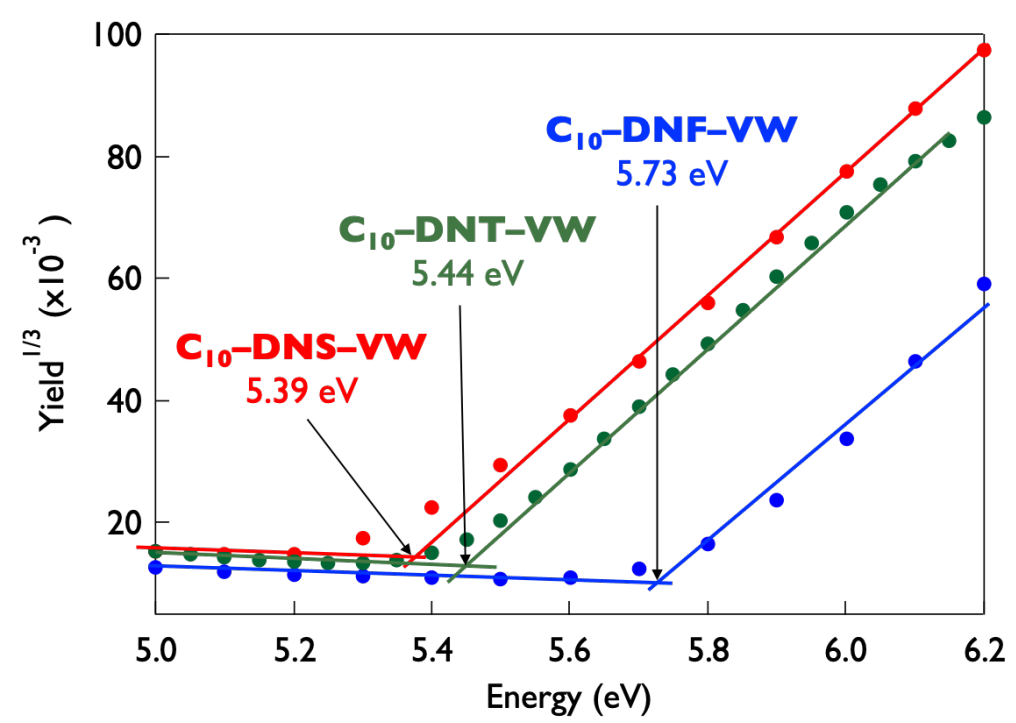

Figure S4. Photoelectron yield spectra of $\mathbf{C}_{\mathbf{1 0}}-\mathrm{DNF}-\mathrm{VW}, \mathrm{C}_{\mathbf{1 0}}-\mathrm{DNT}-\mathrm{VW}$ and $\mathrm{C}_{\mathbf{1 0}}-\mathrm{DNS}-\mathrm{VW}$ in thin films after light illumination from a D2 lamp in vacuo. 
Table S1. Ionization potentials of unsubstituted and decyl-substituted V-shaped derivatives.

\begin{tabular}{ccc}
\hline Compounds & Ionization potentials $(\mathrm{eV})$ & Calculated HOMO $(\mathrm{eV})^{\mathrm{a}}$ \\
\hline DNF-V & 5.93 & -5.80 \\
DNT-V & 5.72 & -5.62 \\
DNS-V & 5.63 & -5.55 \\
C $_{\mathbf{1 0}}$-DNF-VW & 5.73 & $-5.72^{\mathrm{b}}$ \\
$\mathbf{C}_{\mathbf{1 0}}$-DNT-VW & 5.44 & $-5.54^{\mathrm{b}}$ \\
$\mathbf{C}_{\mathbf{1 0}}$-DNS-VW & 5.39 & $-5.48^{\mathrm{b}}$ \\
\hline
\end{tabular}

${ }^{a}$ HOMO levels were calculated at the B3LYP/6-311G(d)//B3LYP/6-31G(d) level of theory.

${ }^{\mathrm{b}} \mathrm{Me}$-substituted derivatives were applied to use for calculation. 


\section{Single-Crystal Analyses}

Single crystals of DNS-V were obtained by horizontal physical vapor transport (PVT) technique. The structural data were successfully collected by single crystal X-ray diffraction (SC-XRD) analysis (Figure S5). The length and diameter of Pyrex tube used are $120 \mathrm{~cm}$ and $2 \mathrm{~cm}$, respectively. Under an Ar carrier gas flow, source and crystallization-zone were heated at a certain temperature to produce their single crystals. Flow rate of Ar carrier gas and source and crystallization-zone temperature were summarized in Table S2.

a)

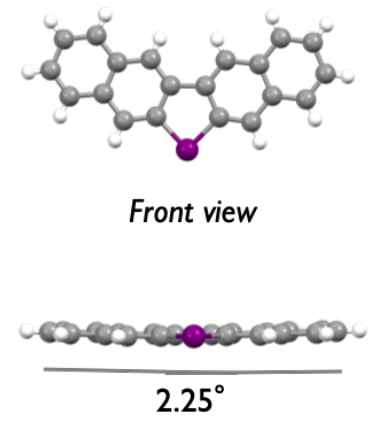

Side view b)

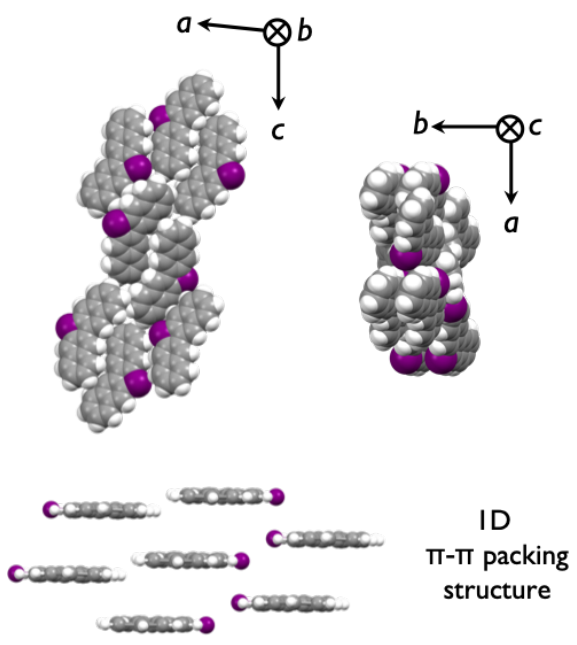

Figure S5. a) Molecular structure and b) aggregated structure of DNS-V in single-crystal structure.

Table S2. PVT conditions for crystallization of DNS-V.

\begin{tabular}{ccc}
\hline $\begin{array}{c}\text { Source temp. } \\
\left({ }^{\circ} \mathrm{C}\right)\end{array}$ & $\begin{array}{c}\text { Crystallization-zone } \\
\text { temp. }\left({ }^{\circ} \mathrm{C}\right)\end{array}$ & $\begin{array}{c}\text { Ar flow rate } \\
(\mathrm{ccm})\end{array}$ \\
\hline 230 & 170 & 50 \\
\hline
\end{tabular}




\section{Differential Scanning Calorimetric (DSC) Analyses}

DSC measurements were carried out with a Rigaku Thermo Plus EVO II DSC8270. Sample was placed in an aluminum pan and heated at the rate of $5 \mathrm{~K} \mathrm{~min}^{-1}$, under $\mathrm{N}_{2}$ purge at a flow rate of 100 $\mathrm{mL} \mathrm{min}{ }^{-1} \cdot \mathrm{Al}_{2} \mathrm{O}_{3}$ was used as the reference material. The DSC data are depicted in Figure S6-7.

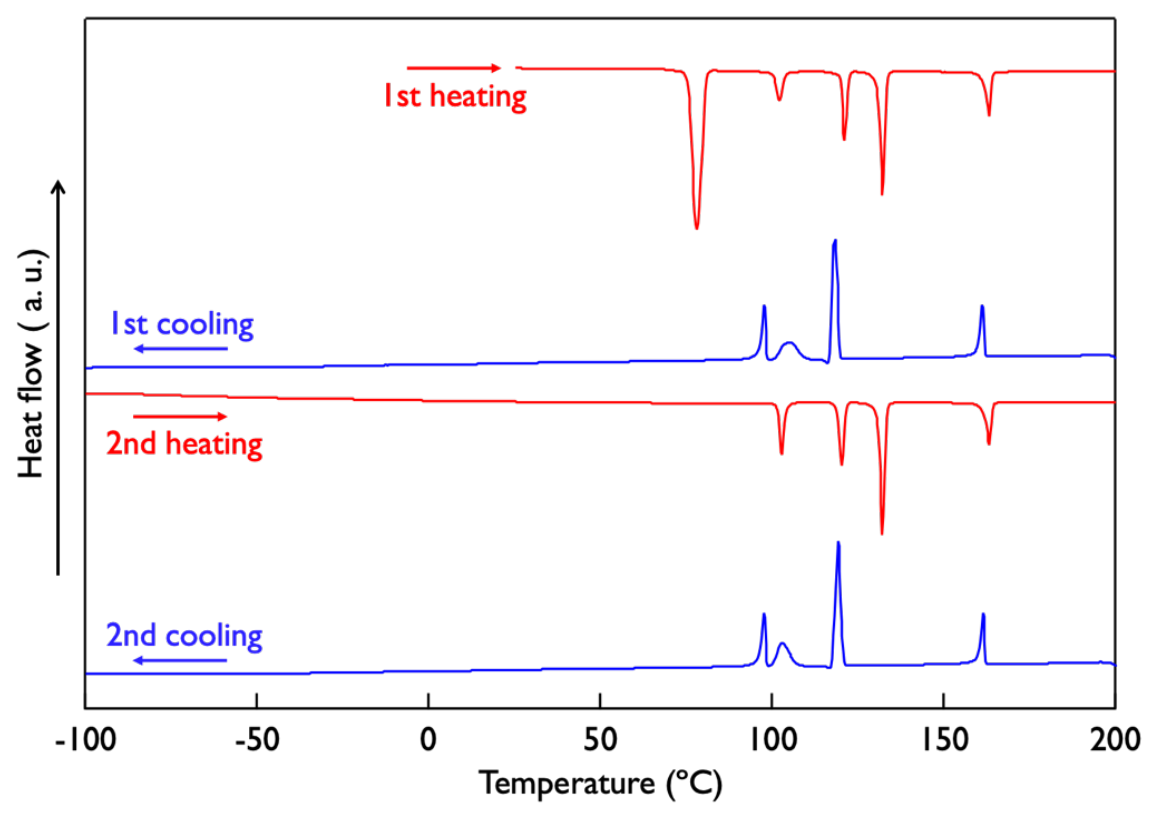

Figure S6. DSC charts of $\mathbf{C}_{10}-\mathbf{D N S}-\mathbf{V W}$ at a scanning rate of $5 \mathrm{~K} \mathrm{~min}^{-1}$.

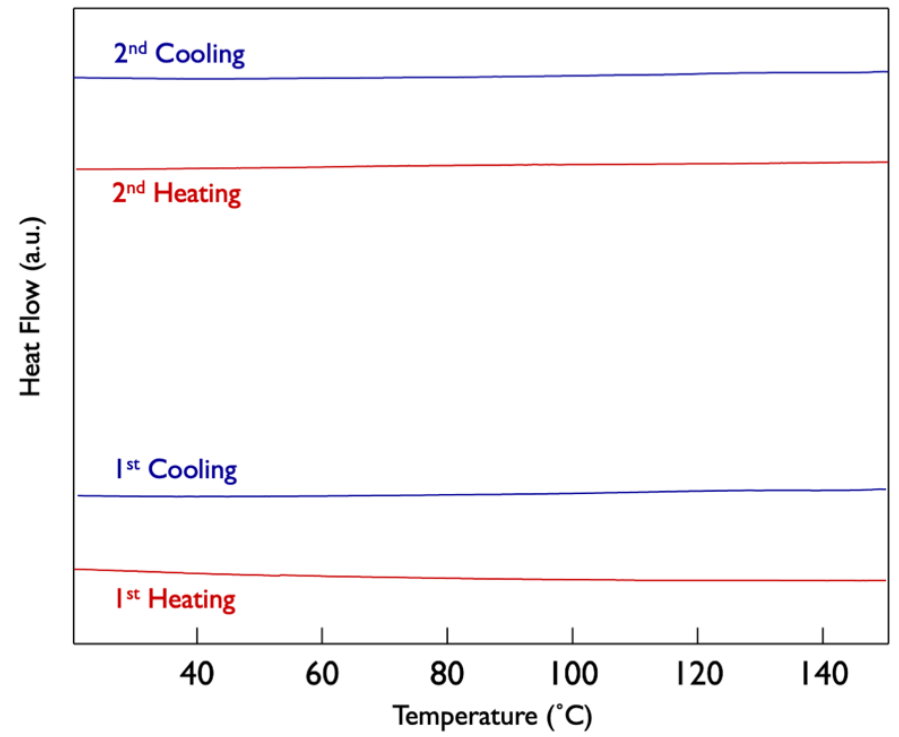

Figure S7. DSC charts of DNS-V at a scanning rate of $5 \mathrm{~K} \mathrm{~min}^{-1}$. 


\section{Powder X-ray Analyses}

A powder X-ray diffraction (PXRD) pattern of $\mathbf{C}_{\mathbf{1 0}}-\mathbf{D N S}-\mathbf{V W}$ was recorded at $303 \mathrm{~K}$ in transmission mode on a diffractometer equipped with an imaging plate detector at the RIKEN Materials Science Beamline (BL44B2) at SPring-8. Measurement conditions were as followed: capillary diameter: $0.3 \mathrm{~mm}$; synchrotron radiation $\lambda=1.08 \AA$; $2 \theta$ range: $1.0-77.58^{\circ}$; step width: $0.01^{\circ} .^{5}$

Phase transition from $\mathbf{C}_{10}-\mathbf{D N S}-\mathbf{V W}$-colorless phase to $\mathbf{C}_{\mathbf{1 0}}-\mathbf{D N S}-\mathbf{V W}$-yellow phase.

The powder sample was prepared as follow. $\mathbf{C}_{\mathbf{1 0}}-\mathbf{D N S}-\mathbf{V W}$ was recrystallized by isopropanol vapor diffusion to the toluene solution to form the colorless crystals. Then, the colorless crystals were carefully crashed and encapsulated in the glass capillary under vacuum. A PXRD pattern of the obtained sample at $30^{\circ} \mathrm{C}$ was confirmed to be in good agreement with the simulated powder pattern of $\mathbf{C}_{10}$-DNS-VW-colorless phase. A completely different diffraction pattern was observed in the heating process at $98{ }^{\circ} \mathrm{C}$ (at a rate of $10 \mathrm{~K} \mathrm{~min}^{-1}$ ), indicating the phase transition from $\mathbf{C}_{\mathbf{1 0}}$-DNS-VW-colorless phase to $\mathbf{C}_{\mathbf{1 0}}-\mathbf{D N S}-\mathbf{V W}$-yellow phase (Figure S8). During the subsequent cooling process to $30^{\circ} \mathrm{C}$, no drastic change was observed. Note that a weak diffraction peak was detected at $2.64^{\circ}$ showing the existence of small amount of $\mathbf{C}_{\mathbf{1 0}}$-DNS-VW-colorless, whereas most of the sample remained in the yellow crystal phase.

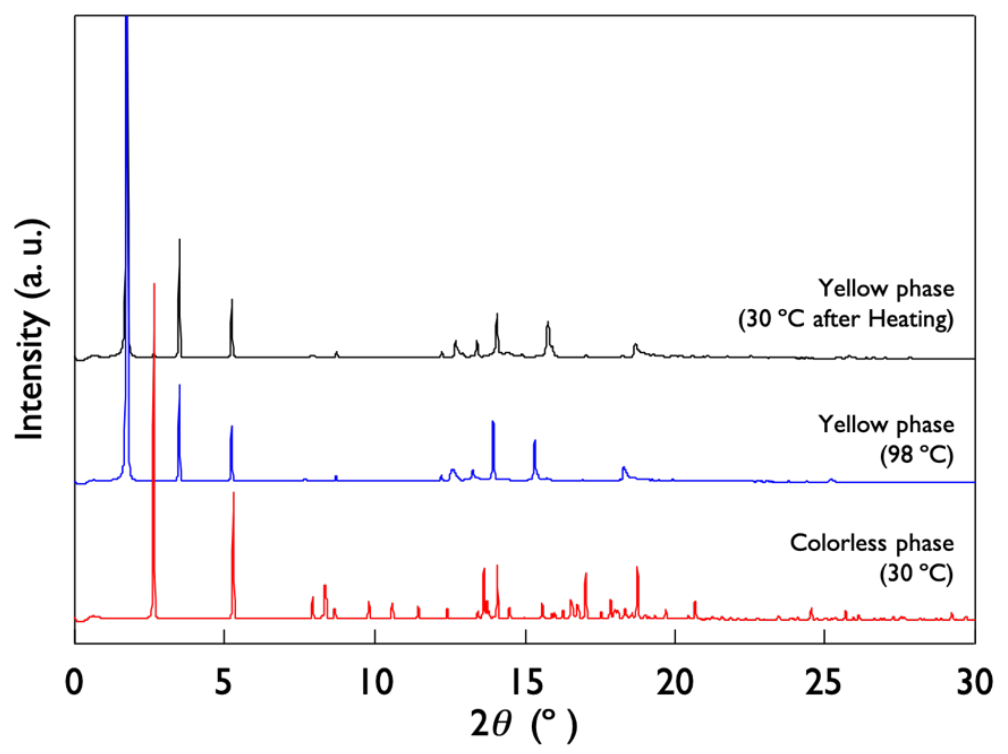

Figure S8. PXRD patterns of $\mathbf{C}_{\mathbf{1 0}}-\mathbf{D N S}-\mathbf{V W}$ : (Bottom) The initial colorless phase at $30^{\circ} \mathrm{C}$; (middle) yellow phase at $98{ }^{\circ} \mathrm{C}$ in the heating process; and (top) the yellow phase at $30^{\circ} \mathrm{C}$ in the subsequent cooling process. 
Variant temperature PXRD measurement and the liquid crystallinity of $\mathbf{C}_{10}-$ DNS-VW.

The colorless crystals were carefully crashed and encapsulated in the glass capillary under vacuum. The capillary was heated to $98{ }^{\circ} \mathrm{C}$ at a rate of $10 \mathrm{~K} \mathrm{~min}^{-1}$ to transform the colorless crystals to the yellow crystals. The PXRD measurements were performed in the range of $30^{\circ} \mathrm{C}$ to $180{ }^{\circ} \mathrm{C}$ in the heating process (Figure S9) and in the subsequent cooling process (Figure S10) at a rate of $10 \mathrm{~K}$ $\mathrm{min}^{-1}$. In Figure S9 and S10, many diffraction peaks were observed below $125{ }^{\circ} \mathrm{C}$, indicating the formation of crystalline phases in the temperature range. However, only one diffraction peak at $1.78^{\circ}$ and broad peak around $13.7^{\circ}$ were obtained at $145^{\circ} \mathrm{C}$, showing that $\mathbf{C}_{\mathbf{1 0}}-\mathbf{D N S}-\mathbf{V W}$ exhibits a liquid crystalline phase in the high temperature region. Polarizing optical microscopic observation supports the formation of smectic A phase in the temperature region (Figure S11). On further heating, a phase transition to the isotropic liquid phase occurs because no obvious peaks were detected at $180^{\circ} \mathrm{C}$.

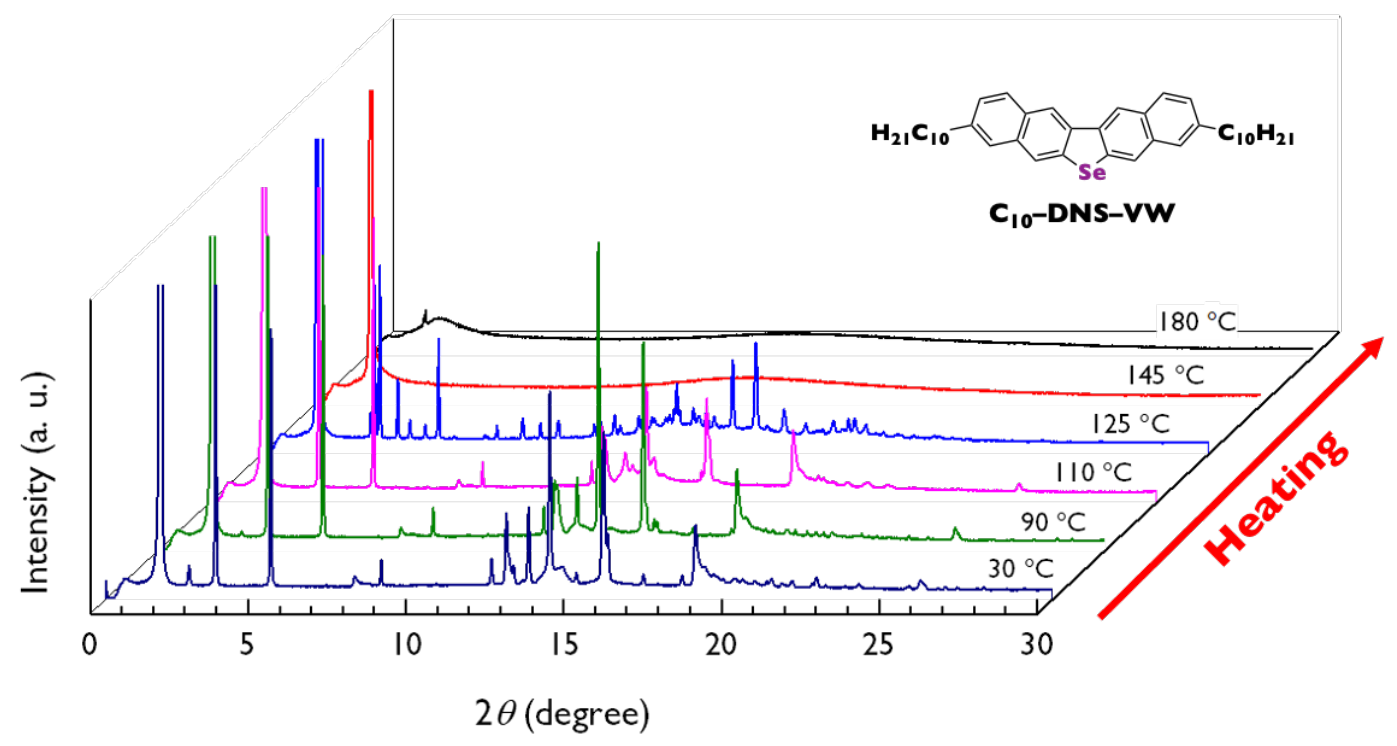

Figure S9. PXRD patterns of $\mathbf{C}_{\mathbf{1 0}}-\mathbf{D N S}-\mathbf{V W}$ in the heating process at a rate of $10 \mathrm{~K} \mathrm{~min}^{-1}$. 


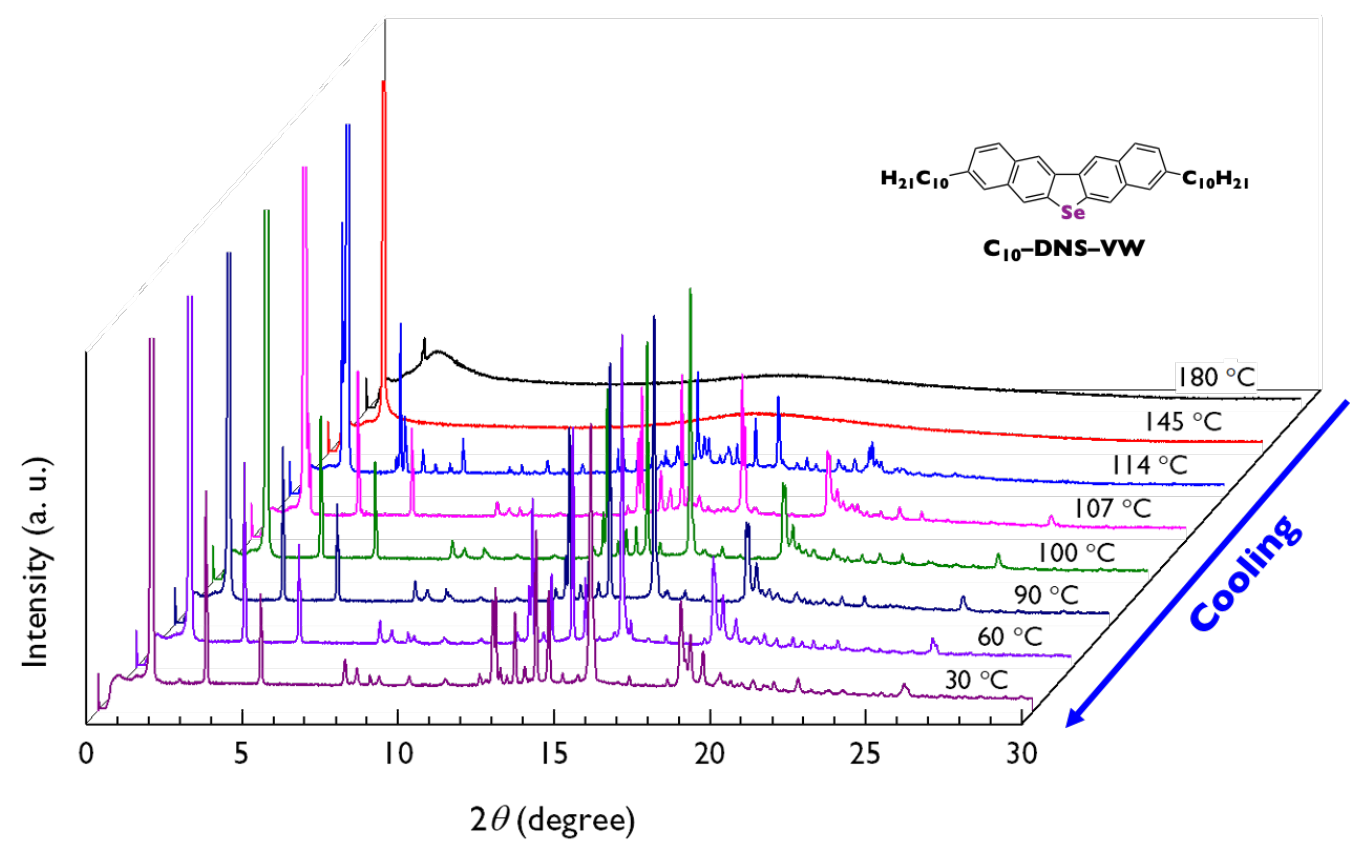

Figure S10. PXRD patterns of $\mathbf{C}_{\mathbf{1 0}}-\mathbf{D N S}-\mathbf{V W}$ in the cooling process at a rate of $10 \mathrm{~K} \mathrm{~min}^{-1}$.
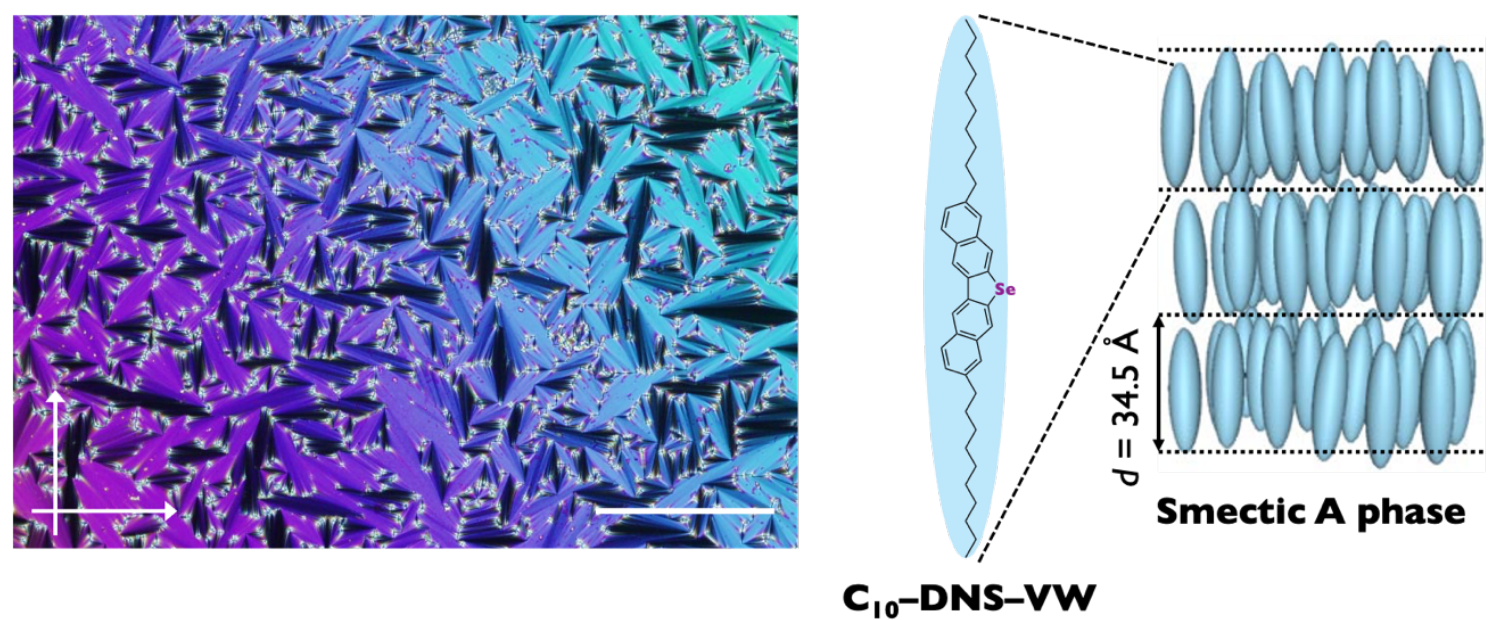

Figure S11. (Left) A polarizing optical microscopic image of $\mathbf{C}_{\mathbf{1 0}}-\mathbf{D N S}-\mathbf{V W}$ at $140{ }^{\circ} \mathrm{C}$ in the smectic A phase. Arrows indicate the directions of polarizer and analyzer axes. Scale bar: $200 \mu \mathrm{m}$. (right) A molecular structure of $\mathbf{C}_{\mathbf{1 0}}-\mathbf{D N S}-\mathbf{V W}$ and schematic illustration of the smectic A phase. 
Vapor-induced phase transition from $\mathbf{C}_{10}-\mathbf{D N S}-\mathbf{V W}$-yellow phase to $\mathbf{C}_{10}-\mathbf{D N S}-\mathbf{V W}$-colorless phase.

As an initial sample, $\mathbf{C}_{\mathbf{1 0}}$-DNS-VW was recrystallized by IPA vapor diffusion to the toluene solution to form the colorless crystals, which were subsequently carefully crashed. Next, to obtain the sample in the yellow phase, the colorless crystals were heated to $98^{\circ} \mathrm{C}$ at a rate of $10 \mathrm{~K} \mathrm{~min}^{-1}$. Finally, to recover the colorless phase, the yellow sample was exposed to the saturated $\mathrm{CHCl}_{3}$ vapor at room temperature for overnight. The three samples obtained in each crystalline phase were encapsulated in the glass capillary under vacuum and PXRD measurements were performed for the three samples (Figure S12).
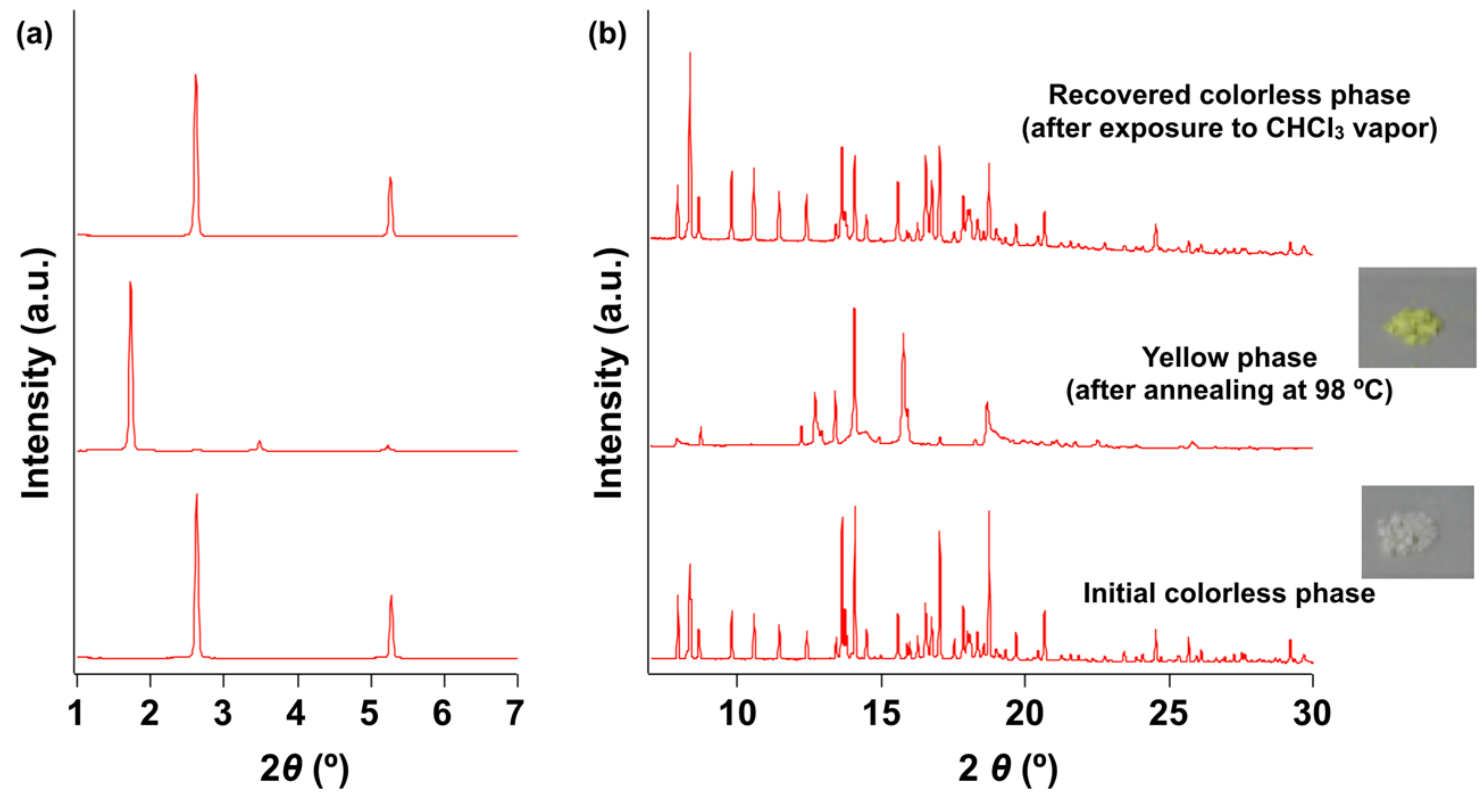

Figure S12. PXRD patterns of $\mathbf{C}_{10}-\mathbf{D N S}-\mathbf{V W}$ : (a) in the small angle region; (b) wide angle region obtained at $30{ }^{\circ} \mathrm{C}$ using synchrotron radiation. (Bottom) the initial colorless phase, (middle) the yellow phase after heating, and (top) the recovered colorless phase after exposure to the $\mathrm{CHCl}_{3}$ vapor. 


\section{Powder UV-vis Absorption Spectra.}

Powder UV-vis absorption spectra of $\mathbf{C}_{\mathbf{1 0}}-\mathbf{D N S}-\mathbf{V W}$-colorless and -yellow were measured in $\mathrm{KBr}$ matrix, along with $\mathbf{C}_{\mathbf{1 0}}-\mathbf{D N S}-\mathbf{V W}$ in 1,2-dichloroethane in comparison (Figure S13).

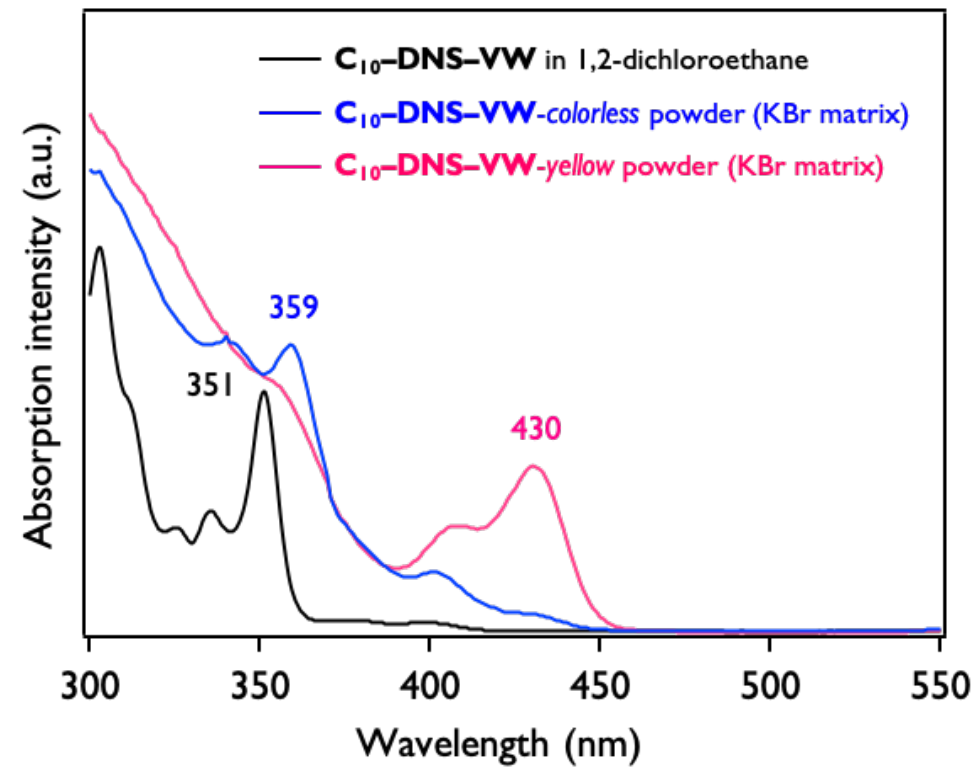

Figure S13. Powder UV-vis absorption spectra of $\mathbf{C}_{\mathbf{1 0}}-\mathbf{D N S}-\mathbf{V W}$ in $\mathrm{KBr}$ matrix. 


\section{UV-vis and Time-Dependent UV-vis Absorption Spectra in Solution and Deposited Thin}

Film

UV-vis absorption spectra were measured with a JASCO V-570 spectrometer. Samples in solution were prepared in degassed 1,2-dichloroethane. Thin films were prepared by vacuum deposition with the thickness of $c a .100 \mathrm{~nm}$ on quartz substrates. The prepared samples in solution and thin films were stored under ambient atmosphere (room temperature and room light) between measurements. Spectra had not change over at least 2 weeks for solution (Figure S14) and 4 weeks for deposited film (Figure S15), which indicates $\mathbf{C}_{\mathbf{1 0}}-\mathbf{D N S}-\mathbf{V W}$ is chemically stable in both solution and solid state.

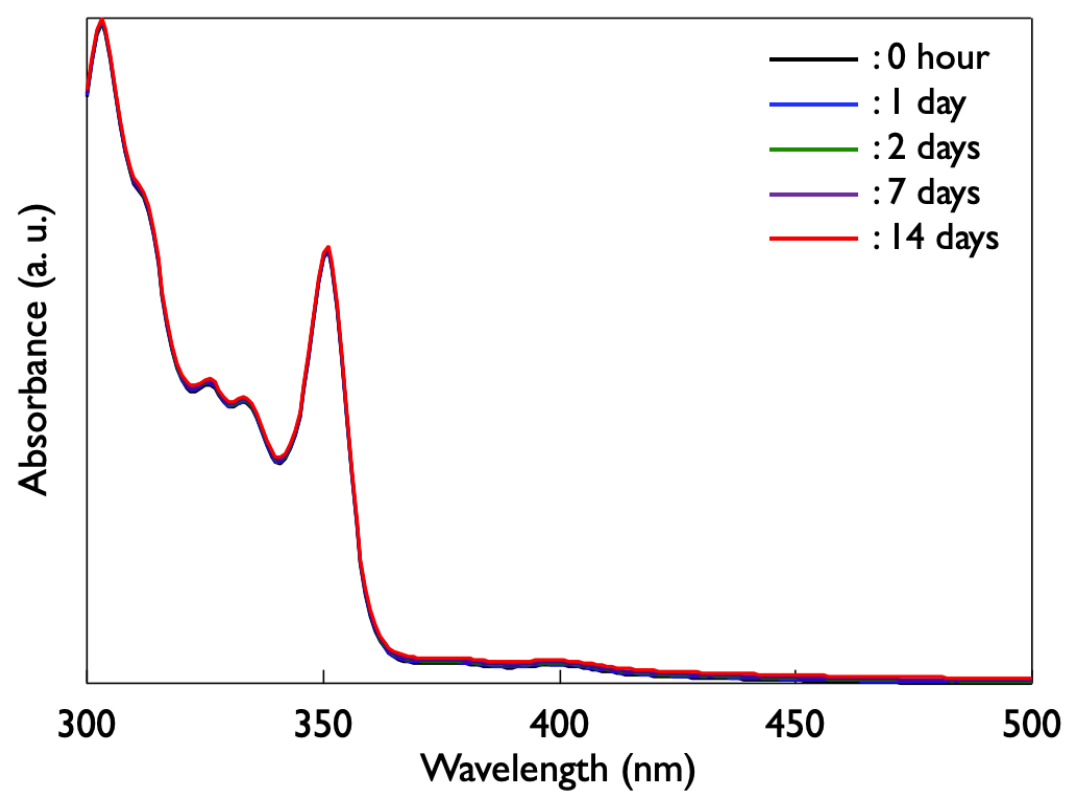

Figure S14. Time-dependent absorption spectra of $\mathbf{C}_{\mathbf{1 0}} \mathbf{- D N S}-\mathbf{V W}$ in 1,2-dichloroethane. 


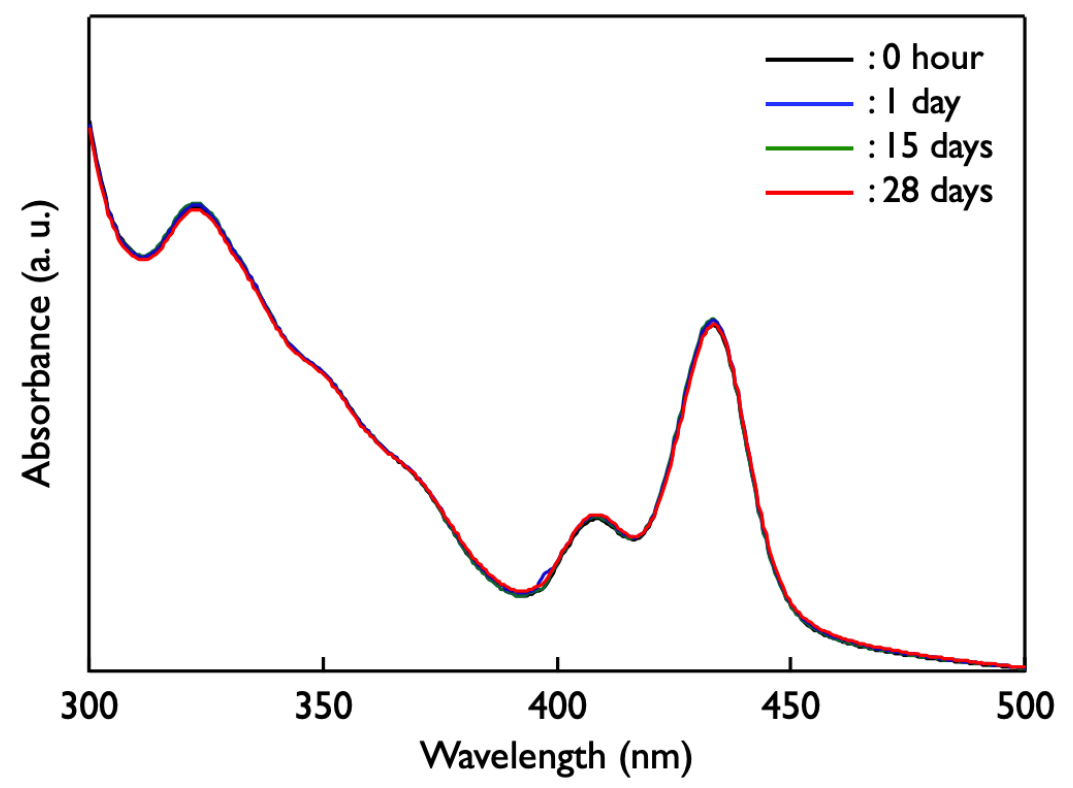

Figure S15. Time-dependent absorption spectra of a ca.100-nm-thick deposited film of C10-DNS-VW. 


\section{Theoretical Calculations}

\subsection{LUMO, HOMO, and NHOMO Energy Levels}

Theoretical calculations of lowest unoccupied molecular orbital (LUMO), highest occupied molecular orbital (HOMO), next highest occupied molecular orbitals (NHOMO) for unsubstituted V-shaped derivatives (DNF-V, DNT-V and DNS-V) were conducted for a single molecule at the B3LYP/6-31G(d), 6-311G(d), cc-pVDZ, and cc-pVTZ levels of theory using the SPARTAN 16 package, Wavefunction Inc (Table S3).

Table S3. LUMO, HOMO and NHOMO energy levels of DNF-V, DNT-V and DNS-V, calculated at the B3LYP-functional 6-31G(d), 6-311G(d), cc-pVDZ and cc-pVTZ levels of theory.

\begin{tabular}{|c|c|c|c|c|c|}
\hline \multirow[b]{2}{*}{ Compounds } & \multirow{2}{*}{$\begin{array}{l}\text { LUMO, HOMO and } \\
\text { HOMO-LUMO gap }\end{array}$} & \multicolumn{4}{|c|}{ Energy levels and gaps } \\
\hline & & $\begin{array}{l}\text { B3LYP/ } \\
6-31 \mathrm{G}(\mathrm{d})\end{array}$ & $\begin{array}{c}\text { B3LYP/ } \\
6-311 \mathrm{G}(\mathrm{d})\end{array}$ & $\begin{array}{l}\text { B3LYP/ } \\
\text { cc-pVDZ }\end{array}$ & $\begin{array}{l}\text { B3LYP/ } \\
\text { cc-pVTZ }\end{array}$ \\
\hline \multirow[t]{5}{*}{ DNS-V } & LUMO & -1.63 & -1.90 & -1.85 & -1.95 \\
\hline & HOMO & -5.33 & -5.55 & -5.48 & -5.58 \\
\hline & NHOMO & -5.62 & -5.85 & -5.78 & -5.89 \\
\hline & HOMO-LUMO gap & 3.70 & 3.65 & 3.63 & 3.63 \\
\hline & HOMO-NHOMO gap & 0.29 & 0.30 & 0.30 & 0.30 \\
\hline \multirow[t]{5}{*}{ DNF-V } & LUMO & -1.65 & -1.92 & -1.87 & -1.97 \\
\hline & HOMO & -5.55 & -5.80 & -5.72 & -5.83 \\
\hline & NHOMO & -5.66 & -5.89 & -5.83 & -5.93 \\
\hline & HOMO-LUMO gap & 3.90 & 3.88 & 3.85 & 3.86 \\
\hline & HOMO-NHOMO gap & 0.11 & 0.09 & 0.11 & 0.10 \\
\hline \multirow[t]{5}{*}{ DNT-V } & LUMO & -1.64 & -1.91 & -1.86 & -1.96 \\
\hline & НОМо & -5.41 & -5.62 & -5.56 & -5.64 \\
\hline & NHOMO & -5.63 & -5.87 & -5.80 & -5.90 \\
\hline & HOMO-LUMO gap & 3.77 & 3.71 & 3.70 & 3.68 \\
\hline & HOMO-NHOMO gap & 0.22 & 0.25 & 0.24 & 0.26 \\
\hline
\end{tabular}


9.2 LUMO and HOMO Energy Levels, HOMO-LUMO Gaps and Total Energies of $\mathrm{C}_{10}$-DNS-VW-yellow and $\mathrm{C}_{10}$-DNS-VW-colorless.

Theoretical calculations of LUMO and HOMO energy levels, HOMO-LUMO gaps for C $_{10}$-DNS-VW-yellow- and $C_{10}-$ DNS-VW-colorless-based molecular structures were conducted at the B3LYP/6-311G(d) levels using the SPARTAN 16 package, Wavefunction Inc (Figure S16).

$$
\mathbf{C}_{10} \text {-DNS-VW-yellow }
$$

Total energy : $-2482844.0671 \mathrm{kcal} \mathrm{mol}^{-1}$
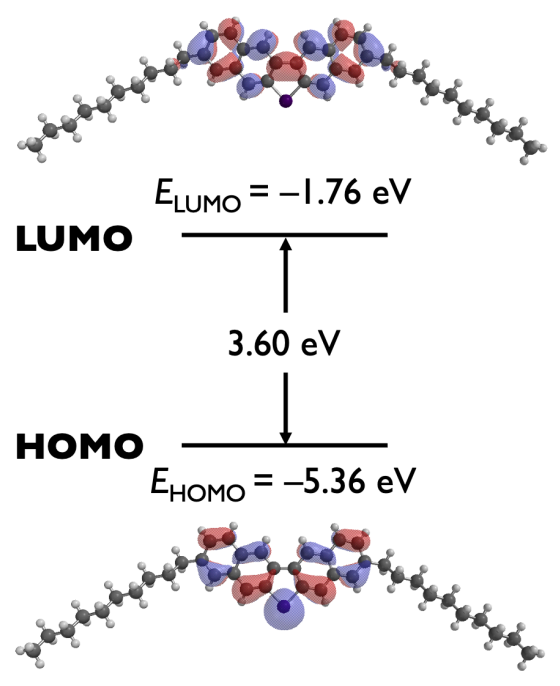

\section{C $_{10}$-DNS-VW-colorless}

Total energy : $-2482925.0095 \mathrm{kcal} \mathrm{mol}^{-1}$ (80.942 kcal mol-1 more stable)

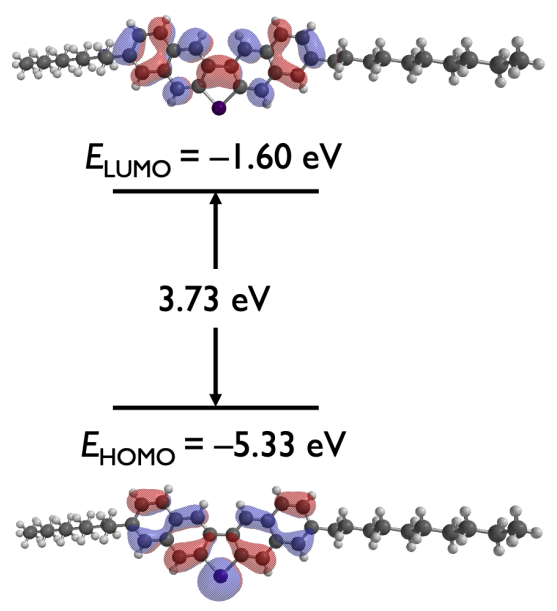

Figure S16. Total energies, LUMO, HOMO and their energy levels, HOMO-LUMO gaps of $\mathbf{C}_{\mathbf{1 0}}$-DNS-VW-yellow and $\mathbf{C}_{\mathbf{1 0}}$-DNS-VW-colorless at the B3LYP/6-311G(d) level of theory. 


\subsection{Intermolecular interaction energy of $\mathrm{C}_{10}-\mathrm{DNS}-\mathrm{VW}$-yellow and $\mathrm{C}_{10}-\mathrm{DNS}-\mathrm{VW}$-colorless.}

Intermolecular interaction energy between two adjacent molecules were obtained at the M06-2X/6-31++G(d,p) level of DFT with counterpoise correction for the basis set superposition error. The calculations were performed using the Gaussian 09 program package (Figure S17).

$$
\text { C }_{10} \text {-DNS-VW-colorless crystal }
$$

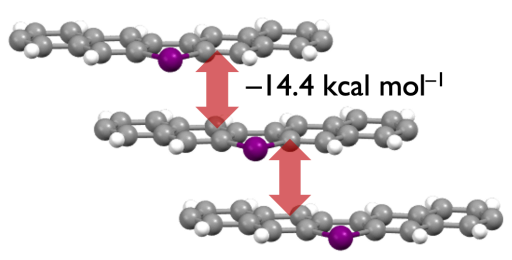

\section{C $_{10}$-DNS-VW-yellow crystal}

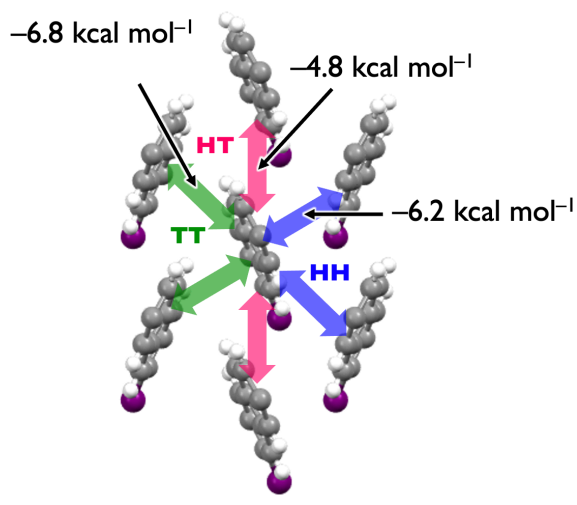

Figure S17. Intermolecular interaction energy of $\mathbf{C}_{\mathbf{1 0}}$-DNS-VW-colorless and C $_{10}$-DNS-VW-yellow between two adjacent molecules. 


\subsection{Transfer Integrals and Band Structure Calculations}

Theoretical calculations of transfer integral and effective mass were conducted using the GAMESS package. ${ }^{6}$ The Kohn-Sham eigenstates of all of compounds in this work were calculated at the PBEPBE/6-31G(d) level of theory. Based on their packing structures, the transfer integrals $(t)$ of HOMO-HOMO and NHOMO-NHOMO of neighboring molecules were estimated by the dimer method. $^{7}$

To further understand the carrier transporting capabilities in the single-crystal state, their HOMO band structures $E(k)$ were also calculated by the tight-binding approximation using transfer integrals. Effective masses of electron were calculated as $m^{*}=\left|\hbar^{2}\left(\partial^{2} E(k) / \partial k^{2}\right)^{-1}\right|$ along respective directions.

Table S4. Summary of transfer integrals of V-shaped derivatives.

\begin{tabular}{|c|c|c|c|c|c|c|c|c|}
\hline \multirow[b]{2}{*}{ Compounds } & \multirow{2}{*}{$\begin{array}{l}\text { Packing } \\
\text { mode and } \\
\text { HB } \\
\text { angles }\left(^{\circ}\right)\end{array}$} & \multicolumn{5}{|c|}{ Transfer integrals, $t(\mathrm{meV})^{\mathrm{a}}$} & \multicolumn{2}{|c|}{ Effective masses } \\
\hline & & $t_{\mathrm{HT}}$ & $t_{\mathrm{HH}}$ & $t_{\mathrm{TT}}$ & $t_{1}$ & $t_{2}$ & $\begin{array}{l}m_{\|} * / m_{0} * \\
m_{\perp} * / m_{0} *\end{array}$ & $\begin{array}{l}m_{\mathrm{b}} * / m_{0} *, \\
m_{\mathrm{a}} * / m_{0} * \mathrm{c}\end{array}$ \\
\hline$C_{10}-D N F-V W$ & $\mathrm{HB}, 48.1^{\circ}$ & $\begin{array}{l}-1.4^{\mathrm{b}} \\
-17.5^{\mathrm{c}}\end{array}$ & $\begin{array}{l}-2.5^{\mathrm{b}} \\
-76.4^{\mathrm{c}}\end{array}$ & $\begin{array}{l}-15.4^{\mathrm{b}} \\
-77.8^{\mathrm{c}}\end{array}$ & - & - & $5.2,1.8$ & - \\
\hline $\mathrm{C}_{10}-\mathrm{DNT}-\mathrm{VW}$ & $\mathrm{HB}, 49.2^{\circ}$ & $\begin{array}{l}+17.6^{\mathrm{b}} \\
+45.2^{\mathrm{c}}\end{array}$ & $\begin{array}{l}-32.4^{\mathrm{b}} \\
-36.7^{\mathrm{c}}\end{array}$ & $\begin{array}{l}-13.7^{b} \\
-60.6^{c}\end{array}$ & - & - & $1.5,2.7$ & - \\
\hline $\begin{array}{c}\mathbf{C}_{10}-\mathbf{D N S}-\mathbf{V W} \\
- \text { yellow phase }\end{array}$ & $\mathrm{HB}, 46.2^{\circ}$ & $\begin{array}{l}+60.8^{\mathrm{b}} \\
+18.4^{\mathrm{c}}\end{array}$ & $\begin{array}{l}-61.8^{\mathrm{b}} \\
-11.9^{\mathrm{c}}\end{array}$ & $\begin{array}{l}-25.0^{\mathrm{b}} \\
-20.8^{\mathrm{c}}\end{array}$ & - & - & $1.2,3.5$ & - \\
\hline $\begin{array}{l}\mathbf{C}_{\mathbf{1 0}} \text {-DNS-VW } \\
\text {-colorless phase }\end{array}$ & $\begin{array}{c}\text { 1D } \\
\pi \text {-stacking }\end{array}$ & - & - & - & $\begin{array}{l}-96.0^{\mathrm{b}} \\
-61.5^{\mathrm{c}}\end{array}$ & $\begin{array}{l}+0.7^{\mathrm{b}} \\
+0.7^{\mathrm{c}}\end{array}$ & - & $>6,1.9$ \\
\hline
\end{tabular}

\footnotetext{
a Transfer integrals are calculated at the PBEPBE/6-31G(d) level of theory.

b Transfer integrals between HOMOs.

c Transfer integrals between NHOMOs.
} 

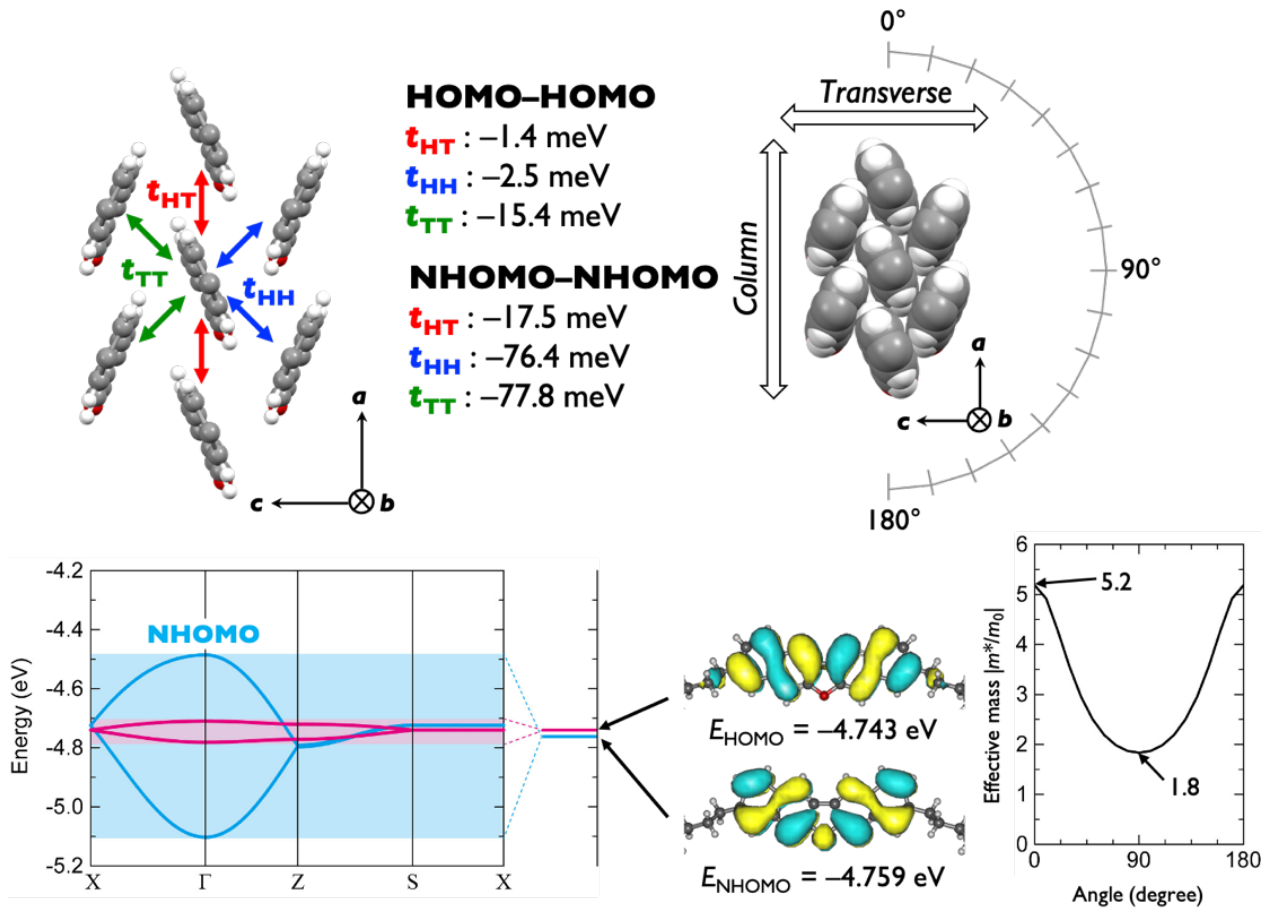

Figure S18. Packing structure of $\mathbf{C}_{\mathbf{1 0}}-\mathbf{D N F}-\mathbf{V W}$ along with transfer integrals of HOMO-HOMO and NHOMO-NHOMO, electronic band structure of $\mathbf{C}_{\mathbf{1 0}}-\mathbf{D N F}-\mathbf{V W}$ crystal ( $a-c$ plane) along with $E_{\mathrm{HOMO}}$ and $E_{\mathrm{NHOMO}}$, and effective masses of $\mathbf{C}_{\mathbf{1 0}}-\mathbf{D N F}-\mathbf{V W}$ crystal ( $a-c$ plane) depending on angles from column direction (calculated at the PBEPBE/6-31G(d) level). 


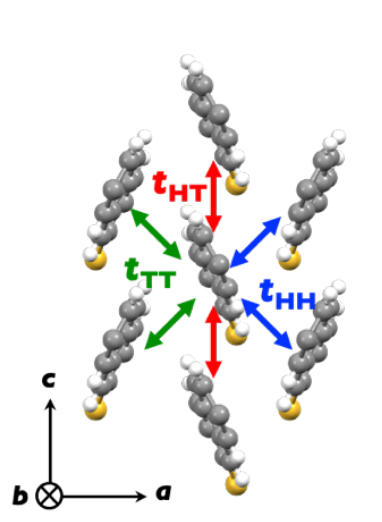

HOMO-HOMO

$t_{\mathrm{HT}}:+17.6 \mathrm{meV}$

$\boldsymbol{t}_{\mathrm{HH}}:-32.4 \mathrm{meV}$

$t_{\mathrm{TT}}:-13.7 \mathrm{meV}$

NHOMO-NHOMO

$t_{\mathrm{HT}}:+45.2 \mathrm{meV}$

$\boldsymbol{t}_{\mathrm{HH}}:-36.7 \mathrm{meV}$

$\boldsymbol{t}_{\mathrm{TT}}:-60.6 \mathrm{meV}$
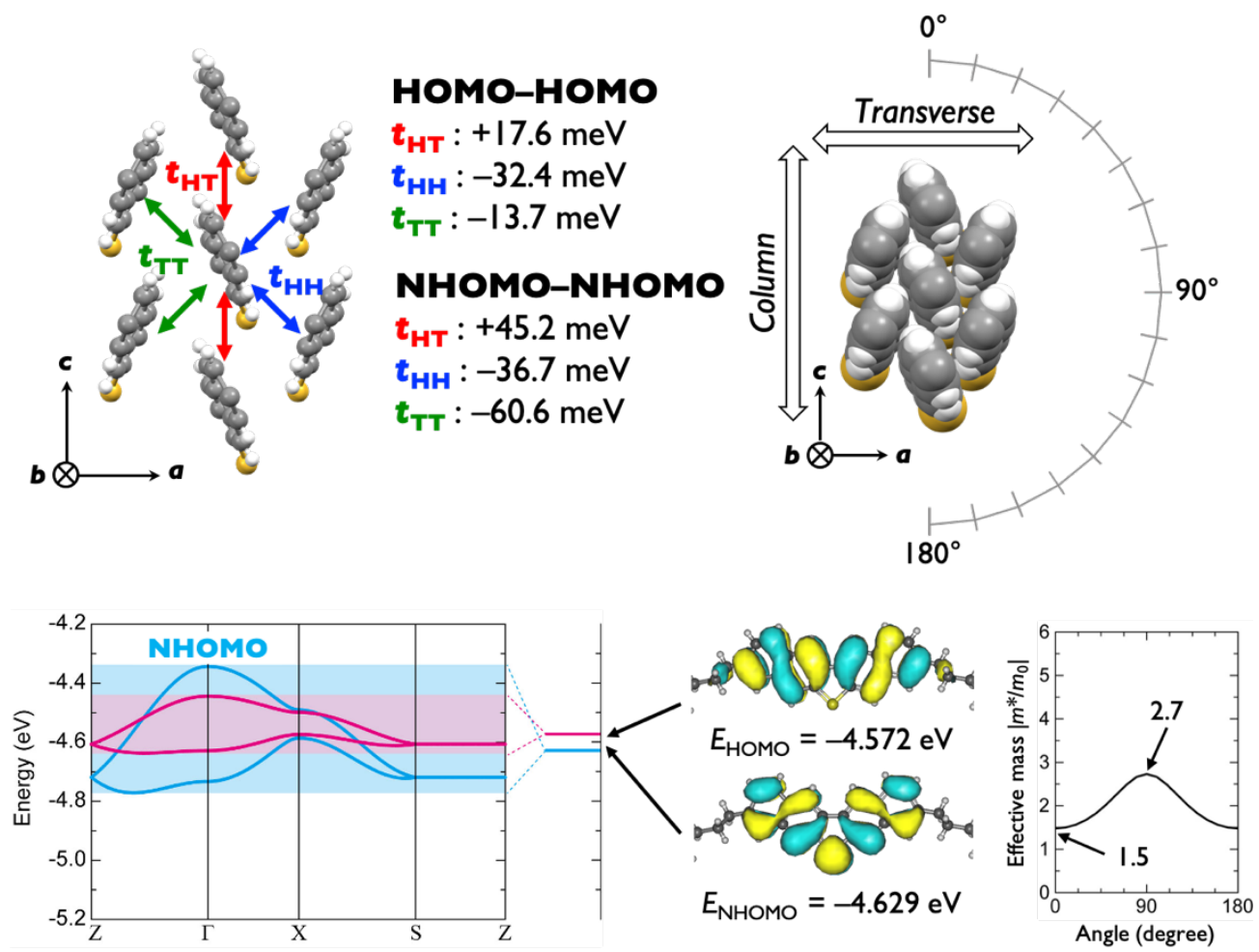

Figure S19. Packing structure of $\mathbf{C}_{\mathbf{1 0}}-\mathbf{D N T}-\mathbf{V W}$ along with transfer integrals of HOMO-HOMO and NHOMO-NHOMO, electronic band structure of $\mathbf{C}_{\mathbf{1 0}}-\mathbf{D N T}-\mathbf{V W}$ crystal ( $a-c$ plane) along with $E_{\mathrm{HOMO}}$ and $E_{\mathrm{NHOMO}}$, and effective masses of $\mathbf{C}_{\mathbf{1 0}}-\mathbf{D N T}-\mathbf{V W}$ crystal ( $a-c$ plane) depending on angles from column direction (calculated at the PBEPBE/6-31G(d) level). 


\section{X-ray Diffraction Measurement and AFM Observation for Polycrystalline Films}

To estimate aggregation structures of vapor-deposited $\mathbf{C}_{\mathbf{1 0}}-\mathbf{D N S}-\mathbf{V W}$ polycrystalline films, the $\mathrm{X}$-ray-diffraction data of vapor-deposited films were collected using Rigaku Mercury 70 with CuK $\alpha$ (wavelength: $1.54056 \AA$ ) radiation. Independent of substrate temperature, the all out-of-plane diffraction patterns give only $\left(\begin{array}{lll}0 & k & 0\end{array}\right)$ peaks under assumption that polycrystalline films have the same aggregation structure as a single crystal of $\mathbf{C}_{\mathbf{1 0}} \mathbf{- D N S}-\mathbf{V W}$-yellow, as shown in Figure S20. Lattice constant was evaluated from a plot of $\sin \theta / \lambda$ vs $k$ to be $70.71 \AA$, which is consistent with $c=$ $70.15 \AA$ in a single crystal of $\mathbf{C}_{\mathbf{1 0}}-\mathbf{D N S}-\mathbf{V W}$-yellow. This result clearly indicates that the polycrystalline film by vapor deposition show herringbone packing structure proper to carrier transport.

a) $T_{\text {sub }}=115^{\circ} \mathrm{C}$

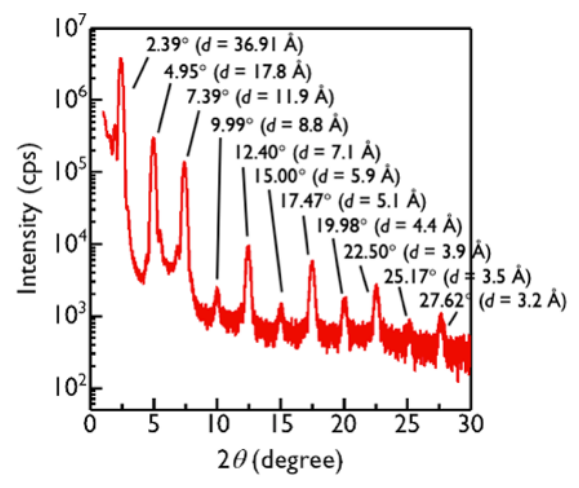

c) $T_{\text {sub }}=\mathrm{RT}$

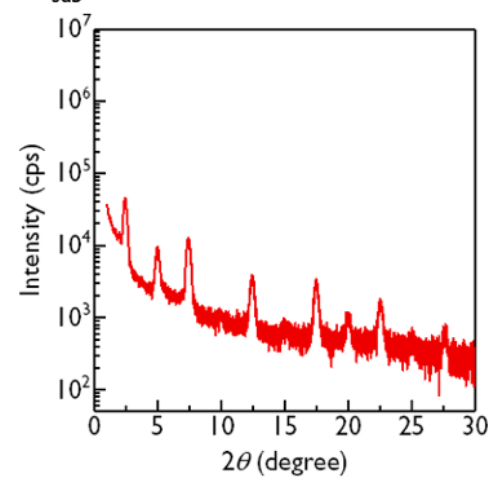

b)

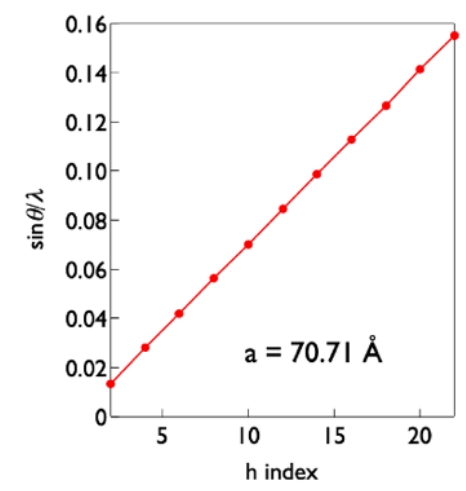

d) $T_{\text {sub }}=90^{\circ} \mathrm{C}$

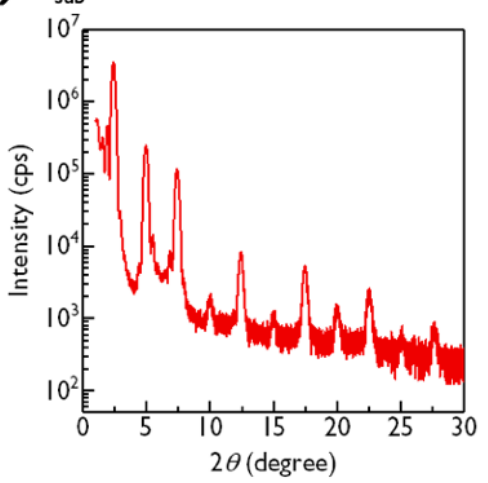

Figure S20. XRD patterns of films deposited at a-b) $115^{\circ} \mathrm{C}$, c) room temperature, and d) $90^{\circ} \mathrm{C}$. 
To observe morphology of the polycrystalline films, atomic force microscopy (AFM) images were taken on a Shimadzu SPM 9700-HT with a non-contact mode. The AFM images are shown in Figure S21. As mentioned above, although aggregation structure is not dependent on substrate temperature, morphology is quite dependent on substrate temperature. The film deposited at $T_{\text {sub }}=$ room temperature has $<500$-nm small grains, and the films deposited at $T_{\text {sub }}=90{ }^{\circ} \mathrm{C}$ and $115^{\circ} \mathrm{C}$ have no obvious grains. Reflected the difference of morphology and grain size, mobility values of the films deposited at room temperature are lower than that of films deposited at $90{ }^{\circ} \mathrm{C}$ and $115{ }^{\circ} \mathrm{C}$. Moreover, heights of steps are consistent with a molecular long axis of $\mathbf{C}_{\mathbf{1 0}}-\mathbf{D N S}-\mathbf{V W}$, which support that the aggregated structures are same as a $\mathbf{C}_{\mathbf{1 0}} \mathbf{- D N S}-\mathbf{V W}$-yellow crystal and $b$ axis is perpendicular to the substrates.

a) $T_{\text {sub }}=115^{\circ} \mathrm{C}$

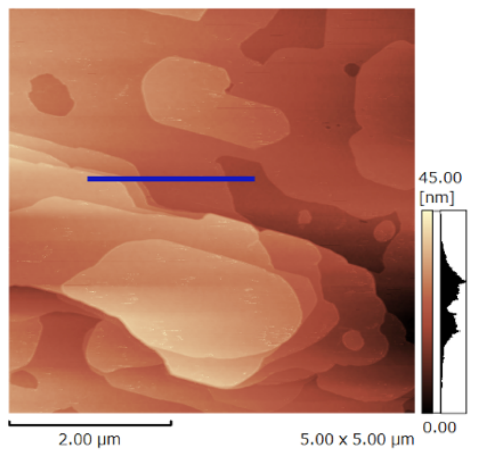

c) $T_{\mathrm{sub}}=\mathrm{RT}$

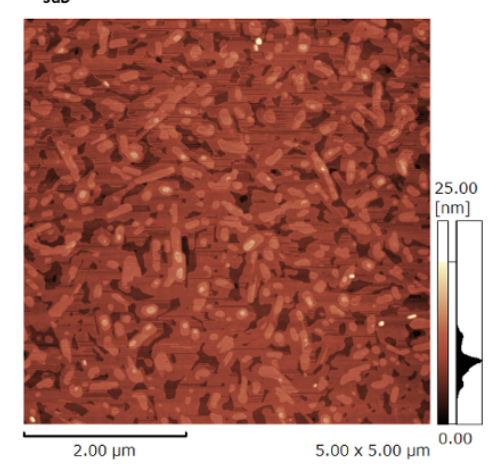

b)

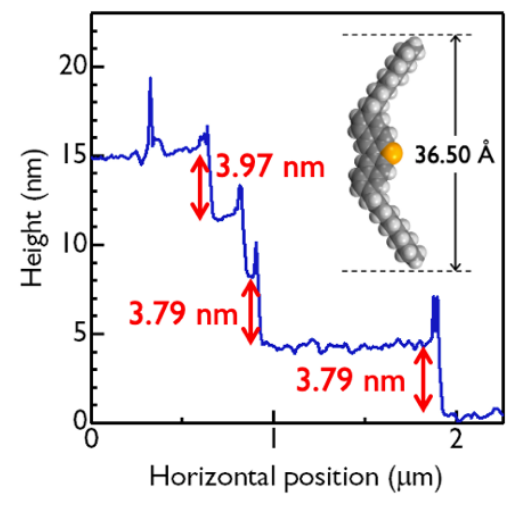

d) $T_{\text {sub }}=90^{\circ} \mathrm{C}$

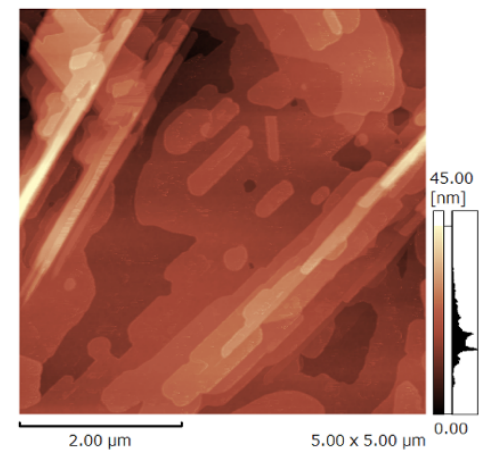

Figure S21. AFM images of films deposited at a) $115^{\circ} \mathrm{C}$, c) room temperature, and d) $90{ }^{\circ} \mathrm{C}$. b) height profile along blue line in a). 


\section{OFET Device Fabrication and Evaluation Procedure}

\subsection{OFET Evaluation}

Transfer and output characteristics of organic field-effect transistors (OFETs) obtained were collected using a semiconductor parameter analyzer (Keithley 4200). Field-effect mobility values $\left(\mu_{\text {sat }}\right)$ were estimated from the saturation regime using the following equation:

$$
-I_{\mathrm{D}}=\left(W C_{\mathrm{i}} / 2 L\right) \mu_{\mathrm{sat}}\left(V_{\mathrm{G}}-V_{\mathrm{th}}\right)^{2}
$$

$C_{\mathrm{i}}$ is the capacitance of gate insulator, $V_{\text {th }}$ is the threshold voltage, and $L$ and $W$ are length and width of the channel, respectively.

\subsection{PVT grown DNS-V single crystal OFET}

The surface of the substrates with 500-nm-thick thermally oxidized $\mathrm{SiO}_{2}$ on doped $\mathrm{Si}$ was washed consecutively with acetone and 2-propanol in an ultra-sound bath for each $5 \mathrm{~min}$, followed by the UV ozone treatment. Then, the cleaned substrates were treated with vapor-deposited heptadecafluorodecyltrimethoxysilane (FDTS). A thin single crystal of DNS-V grown by PVT (see Section 3 in SI) was electrostatically attached to the FDTS-treated substrate. On top of the laminated single crystals, 2,3,5,6-tetrafluoro-7,7,8,8-tetracyanoquinodimethane $\left(\mathrm{F}_{4}-\mathrm{TCNQ}\right)^{8-9}$ as a strong acceptor (thickness: $2 \mathrm{~nm}$ ) to reduce contact resistance and gold electrodes (thickness: $30 \mathrm{~nm}$ ) were successively deposited through the shadow mask to fabricate a bottom-gate-top-contact device. The doped-Si layer acts as a gate electrode and $\mathrm{SiO}_{2}$ layer as gate dielectrics with relative dielectric constant of 3.9.

DNS-V single crystal transistors operate as p-type OFET with negligible hysteresis. Unfortunately, they show large threshold voltage of approximately $-50 \mathrm{~V}$ (on 500-nm-thick $\mathrm{SiO}_{2}$ ). From transfer characteristics in saturation region, mobility is evaluated to be $0.088 \pm 0.013 \mathrm{~cm}^{2} / \mathrm{Vs}$ as an average and $0.11 \mathrm{~cm}^{2} / \mathrm{Vs}$ as maximum (Figure S22). 

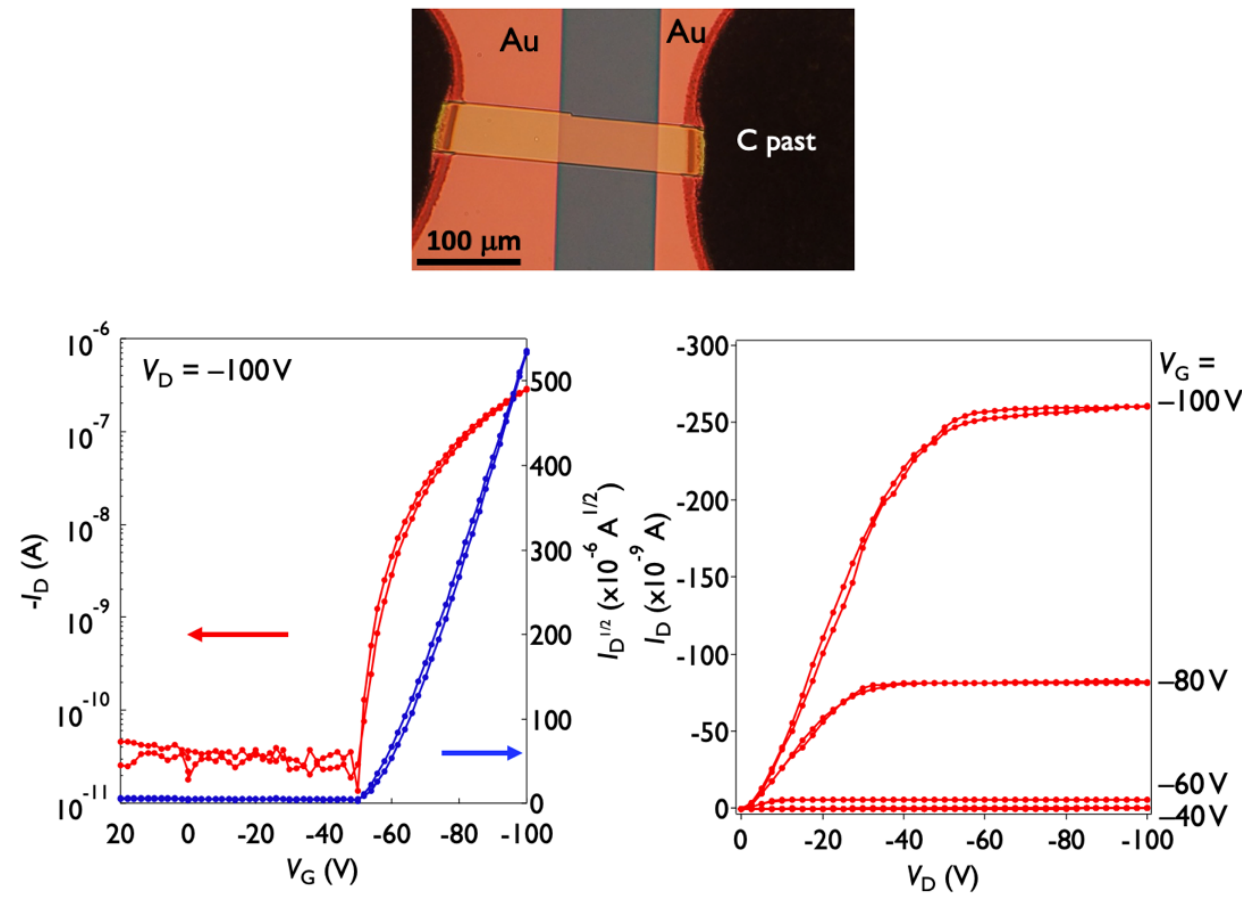

Figure S22. OFET performance of DNS-V single crystal. (top) Top view of the device. The carbon past electrically connected Au electrodes on top of the crystal and on the substrate. (bottom-left) Transfer and (-right) output characteristics $\left(L / W=100 \mu \mathrm{m} / 50 \mu \mathrm{m}, \mathrm{SiO}_{2}: 500 \mathrm{~nm}\right)$.

\subsection{Vapor-deposited C $_{10}$-DNS-VW FET}

The doped $\mathrm{Si}$ substrates with 200-nm-thick $\mathrm{SiO}_{2}$, which surface was treated with decyltrimethoxysilane (DTS) vapor, were consecutively washed with acetone and 2-propanol in an ultra-sound bath. Onto the substrates heated at each temperature $\left(T_{\text {sub }}\right), 40$-nm-thick films of C $_{10}$-DNS-VW were thermally deposited through shadow masks in a vacuum chamber (rate: $0.5 \AA$ $\left.\mathrm{sec}^{-1}\right)$. On the top of the polycrystalline films, $\mathrm{F}_{4}-\mathrm{TCNQ}(2 \mathrm{~nm})^{8-9}$ and gold $(60 \mathrm{~nm})$ as contacts were successively deposited through shadow masks to construct bottom-gate-top-contact architectures.

Transfer and output characteristics of the represented device of each substrate temperature are shown in Figure S23-25. All devices operate as p-type OFETs and slight hysteresis against $V_{\mathrm{G}}$ sweeping. Their average mobility values and maximum values are summarized in Table S5. Their mobility becomes higher in order of $T_{\text {sub }}=$ room temperature, $90^{\circ} \mathrm{C}$, and $115^{\circ} \mathrm{C}$. 

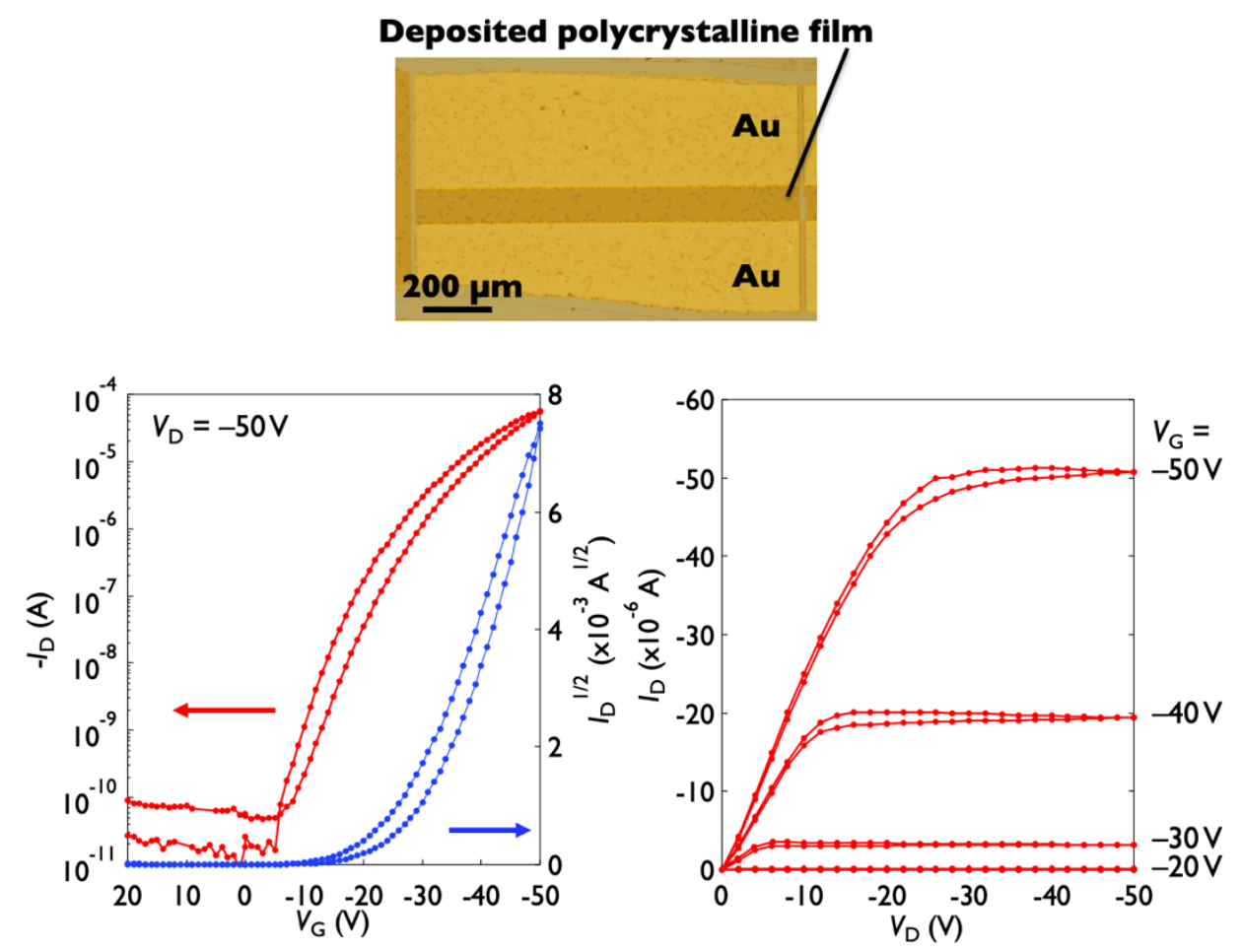

Figure S23. OFET performance of a $\mathbf{C}_{\mathbf{1 0}}-\mathbf{D N S}-\mathbf{V W}$ polycrystalline film deposited at room temperature. (Top) Top view of the device. Transfer (left)- and output (right) characteristics $(L / W=$ $\left.100 \mu \mathrm{m} / 1059 \mu \mathrm{m}, \mathrm{SiO}_{2}: 200 \mathrm{~nm}\right)$.
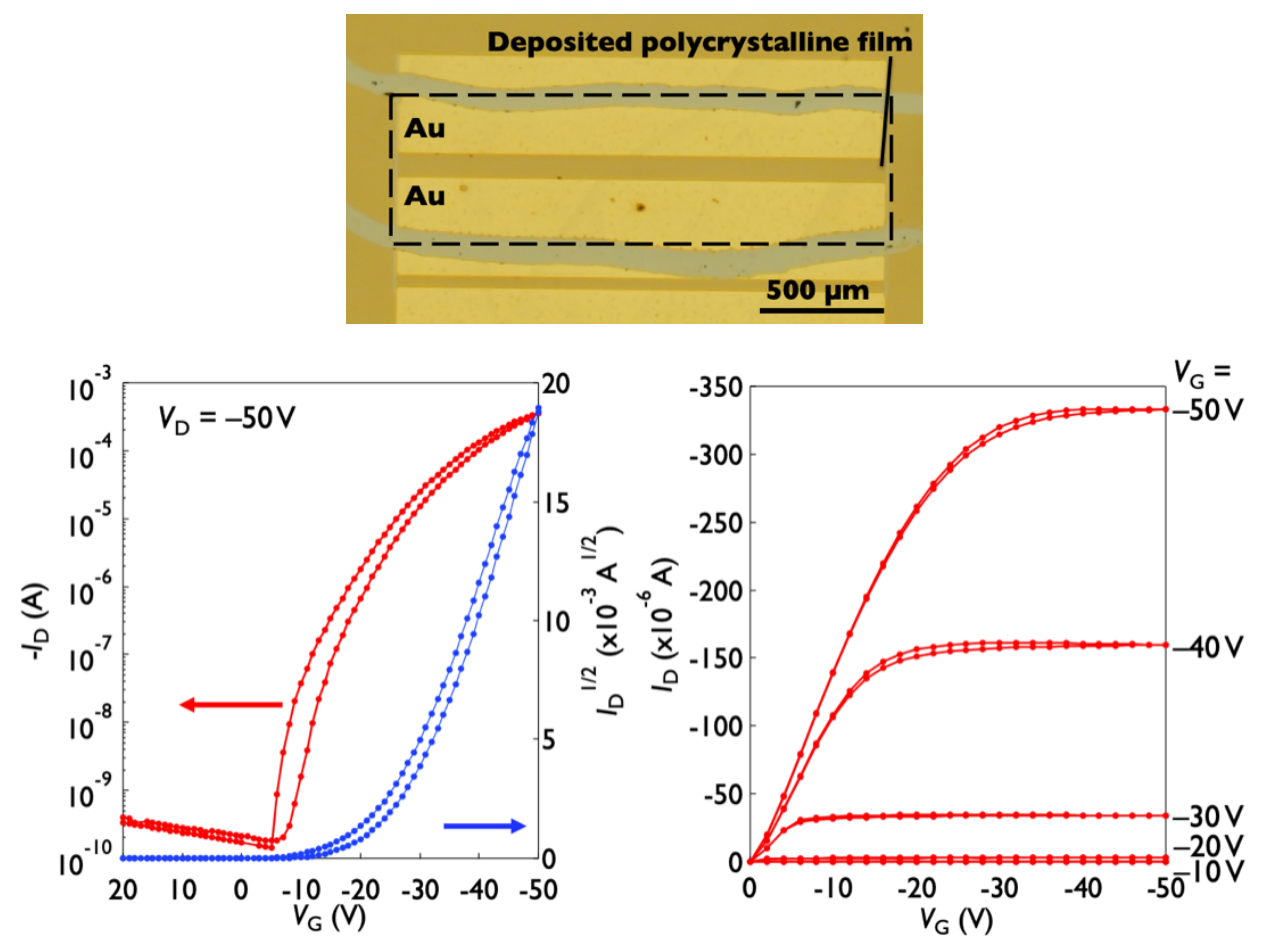

Figure S24. OFET performance of a $\mathbf{C}_{10}-\mathbf{D N S}-\mathbf{V W}$ polycrystalline film deposited at $90{ }^{\circ} \mathrm{C}$. 
Transfer (left)- and output (right) characteristics $\left(L / W=100 \mu \mathrm{m} / 2000 \mu \mathrm{m}, \mathrm{SiO}_{2}: 200 \mathrm{~nm}\right)$.
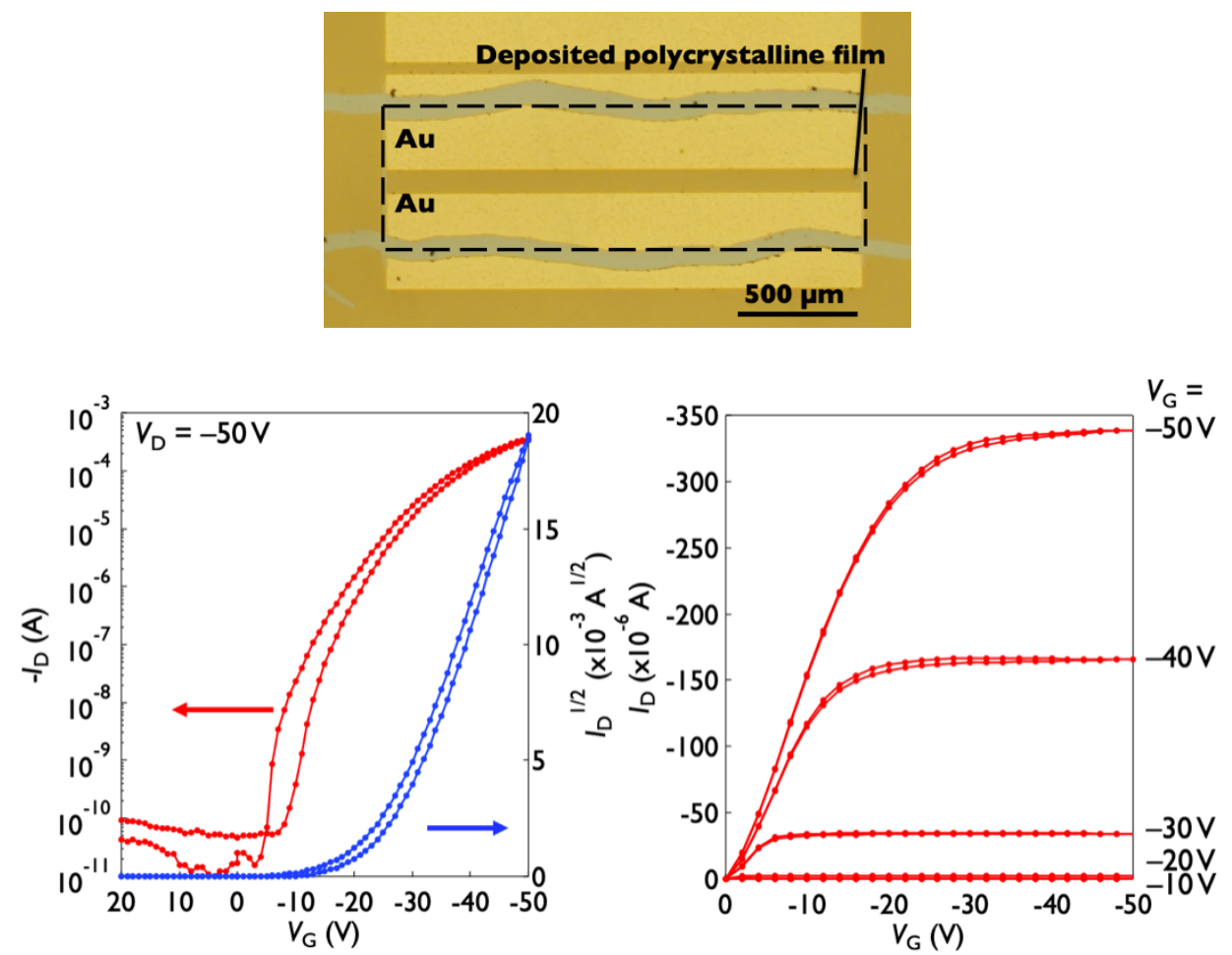

Figure S25. OFET performance of a $\mathbf{C}_{10}-\mathbf{D N S}-\mathbf{V W}$ polycrystalline film deposited at $115^{\circ} \mathrm{C}$. (Top) Top view of the device. Transfer (left)- and output (right) characteristics $(L / W=100 \mu \mathrm{m} / 2000 \mu \mathrm{m}$, $\mathrm{SiO}_{2}: 200 \mathrm{~nm}$ ).

Table S5. Mobility values of $\mathbf{C}_{10}-\mathbf{D N S}-\mathbf{V W}$ polycrystalline films.

\begin{tabular}{ccc}
\hline \multirow{2}{*}{$T_{\text {sub }}\left({ }^{\circ} \mathrm{C}\right)$} & \multicolumn{2}{c}{ Mobility $\left(\mathrm{cm}^{2} / \mathrm{Vs}\right)$} \\
\cline { 2 - 3 } & $\mu_{\text {avg }}{ }^{\mathrm{a}}$ & $\mu_{\max }$ \\
\hline Room temperature & $1.0 \pm 0.05$ & 1.1 \\
$90^{\circ} \mathrm{C}$ & $2.3 \pm 0.6$ & 3.2 \\
$115^{\circ} \mathrm{C}$ & $2.8 \pm 0.4$ & 3.2 \\
\hline
\end{tabular}

a The average mobility is described as an average value along with a standard deviation. 


\subsection{Solution crystallized $\mathrm{C}_{10}$-DNS-VW-yellow FET}

The surface of the cleaned 200-nm-thick thermally oxidized $\mathrm{SiO}_{2}$ on doped $\mathrm{Si}$ substrates is treated with $\beta$-phenylethyltrimethoxysilane ( $\beta$-PTS) in toluene solution. To form the crystalline thin films, $0.01 \mathrm{wt} \%$ anisole solution of $\mathbf{C}_{\mathbf{1 0}}-\mathbf{D N S}-\mathbf{V W}$ was dropped onto the edge of a sustaining glass piece onto the substrates at $40{ }^{\circ} \mathrm{C}$. The crystalline domains grow in the direction of evaporation of the solvent. After the sustaining pieces were removed, the crystalline films were dried at $70{ }^{\circ} \mathrm{C}$ in vacuum for $24 \mathrm{~h}$. Then, $\mathrm{F}_{4}-\mathrm{TCNQ}(2 \mathrm{~nm})^{8-9}$ and gold $(40 \mathrm{~nm})$ as contacts were evaporated on the C $_{10}-\mathbf{D N S}-\mathbf{V W}$ film through a shadow mask. Finally, to determine channel size, unnecessary crystals were removed by laser etching technique.

To observe morphology of the solution-crystallized $\mathbf{C}_{\mathbf{1 0}}$-DNS-VW-yellow thin-film, AFM images were taken on a Shimadzu SPM 9700-HT with a non-contact mode. The AFM images are shown in Figure S26. As can be seen in Figure S26, it exhibits a smooth surface and a gap of $3.7 \mathrm{~nm}$ which corresponds to the molecular length of $\mathbf{C}_{\mathbf{1 0}}-\mathbf{D N S}-\mathbf{V W}$ along the longitudinal axis.
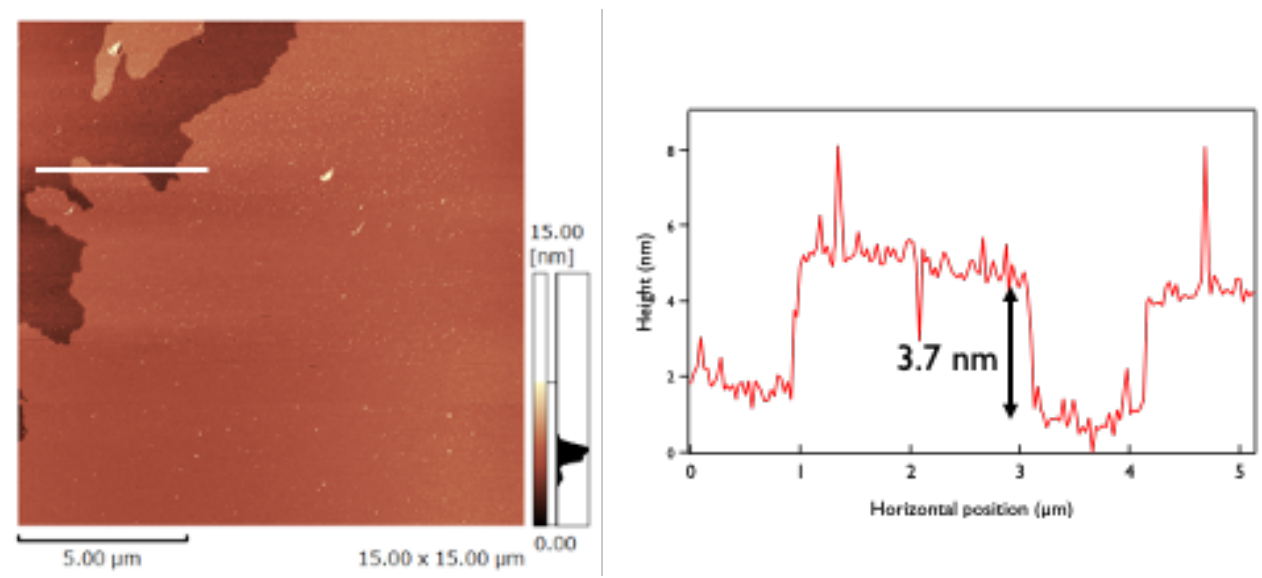

Figure S26. AFM images of the solution-crystallized $\mathbf{C}_{\mathbf{1 0}}$-DNS-VW-yellow thin-film. 


\subsection{Device stability test of solution crystallized $C_{10}-$ DNS-VW-yellow FET against atmospheric stress}

The device was stored in a yellow room (light shielded to $500 \mathrm{~nm}$ or less) at room temperature in the air. Figure S27 shows the output characteristics of a $\mathbf{C}_{\mathbf{1 0}}-\mathbf{D N S}-\mathbf{V W}$-yellow solution-grown single-crystal FET over two months.
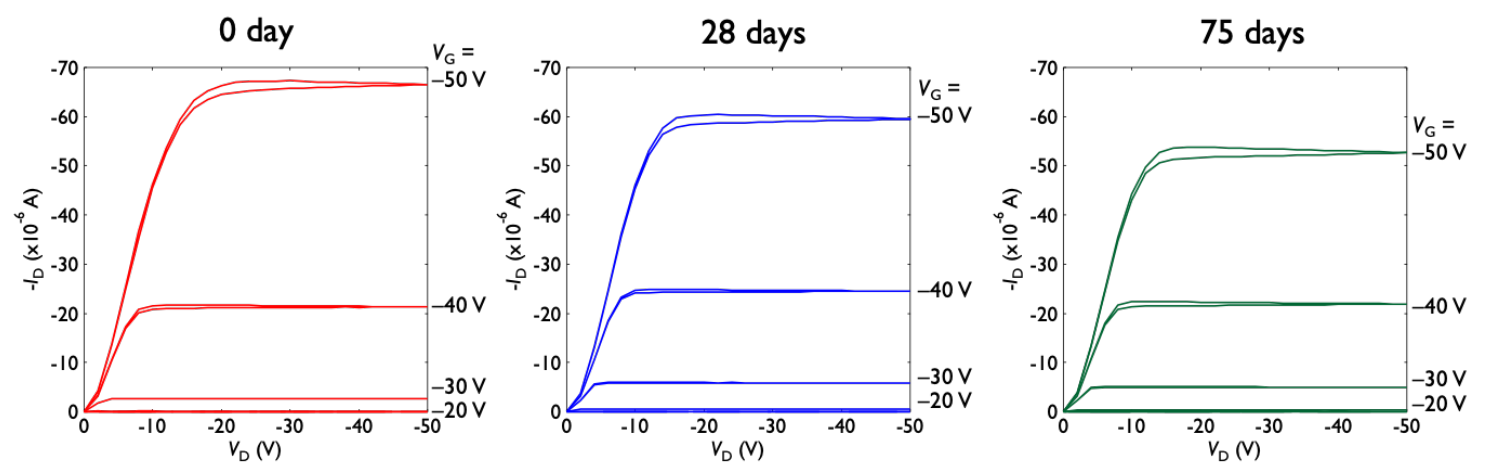

Figure S27. The output characteristics on storage under ambient conditions of a $\mathbf{C}_{\mathbf{1 0}}-\mathbf{D N S}-\mathbf{V W}$ solution-grown single-crystal FET.

\subsection{Single crystal FETs with $\mathrm{C}_{10}-\mathrm{DNS}-\mathrm{VW}$-colorless}

To determine the aggregated structures in thin-films, a $100 \mathrm{~nm}$ thin-film of $\mathbf{C}_{\mathbf{1 0}}-\mathbf{D N S}-\mathbf{V W}$-yellow is grown by vapor deposition and then it is exposed to chloroform vapor. Microscope images are summarized in Figure S28. As can be seen from the image after the treatment, needle-like crystals rather than thin films grew on the substrate. Figure S29 shows the result of XRD data to confirm whether the needle-like crystals after the treatment. The XRD before the exposure showed good agreement with the powder pattern of the $\mathbf{C}_{\mathbf{1 0}}-\mathbf{D N S}-\mathbf{V W}$-yellow form, while the molecules were selectively oriented in the $b$-axis direction on the substrate. On the other hand, the needle-like single crystal grown on the substrate after the vapor treatment showed a good agreement with the powder pattern of the $\mathbf{C}_{10}-\mathbf{D N S}-\mathbf{V W}$-colorless form and also the yellow form is well converted to the colorless one as shown in Figure S29. 


\section{As-deposited}

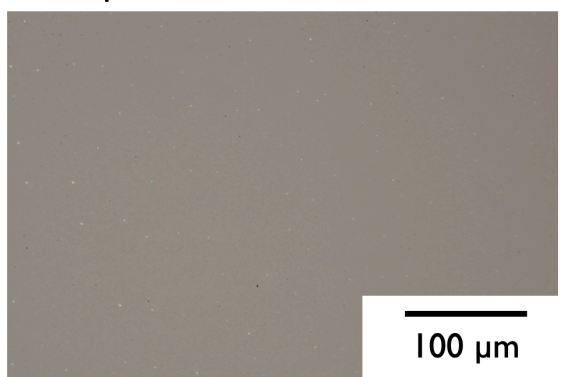

After $\mathrm{CHCl}_{3}$ exposure

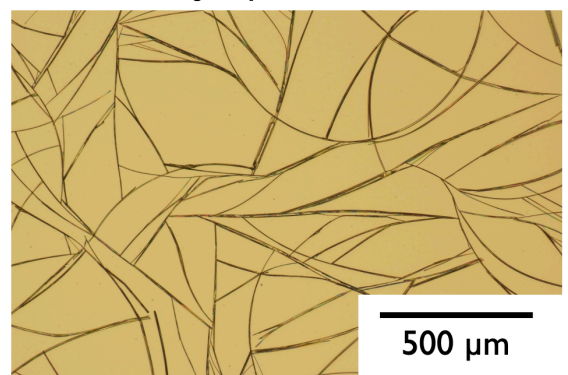

Figure S28. Optical microscope image of vapor-deposited $\mathbf{C}_{\mathbf{1 0}}-\mathbf{D N S}-\mathbf{V W}$ thin film before (as-deposited) and after exposure to $\mathrm{CHCl}_{3}$.

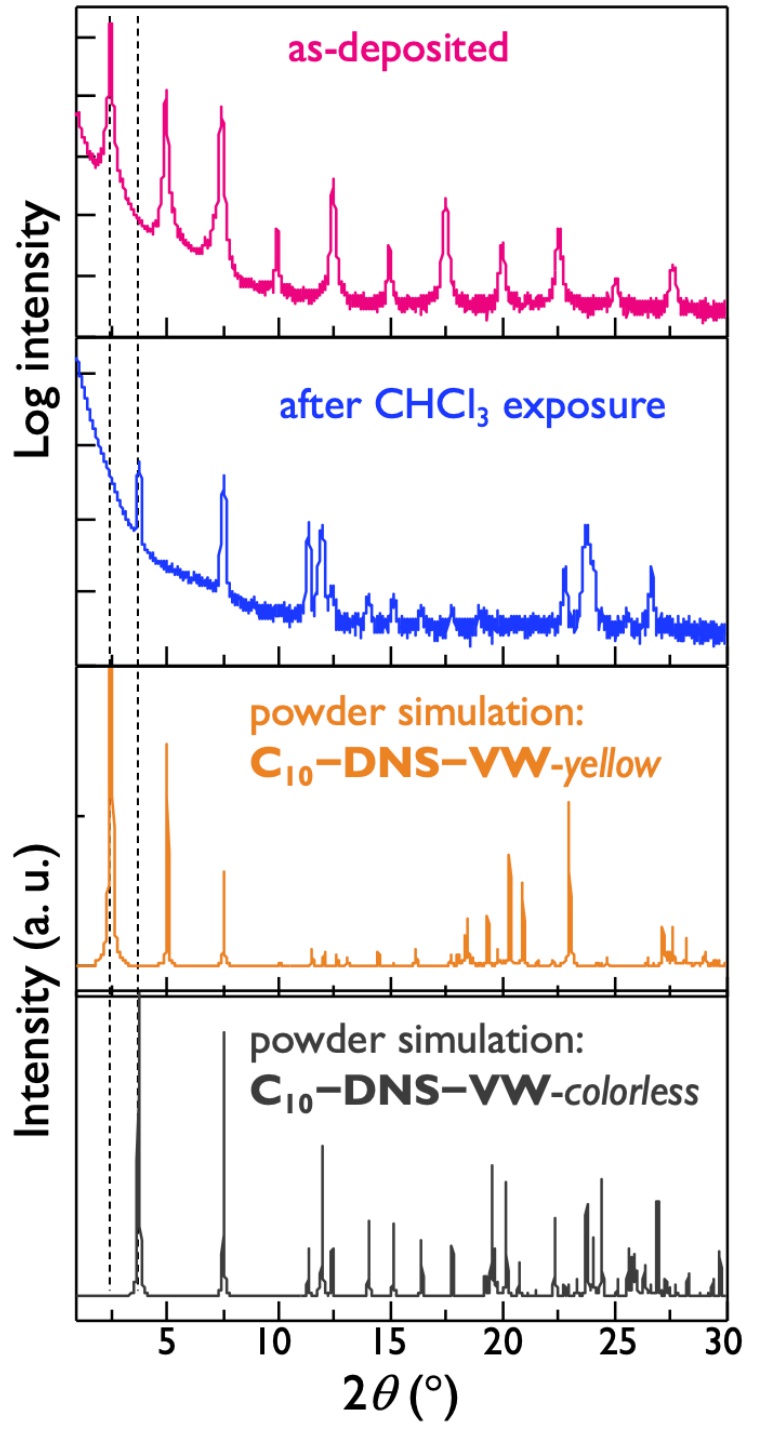

Figure S29. Thin-film XRD patterns of before and after Optical microscope image of 
vapor-deposited $\mathbf{C}_{\mathbf{1 0}}$-DNS-VW thin film before (as-deposited) and after exposure to $\mathrm{CHCl}_{3}$ along with powder simulation of $\mathbf{C}_{\mathbf{1 0}}-\mathbf{D N S}-\mathbf{V W}$-yellow and $\mathbf{C}_{\mathbf{1 0}}-\mathbf{D N S}-\mathbf{V W}$-colorless crystal structures.

To prepare well-ordered single-crystals of $\mathbf{C}_{\mathbf{1 0}}$-DNS-VW-colorless, $\mathbf{C}_{\mathbf{1 0}}$-DNS-VW-yellow crystalline films were fabricated by edge casting method. The dried crystalline film and chloroform were sealed in a glass container at room temperature to expose the film to chloroform vapor overnight. The exposure changed the crystalline thin film ( $\mathbf{C}_{\mathbf{1 0}}-\mathbf{D N S}-\mathbf{V W}$-yellow) into needle crystals ( $\mathbf{C}_{\mathbf{1 0}}-\mathbf{D N S}-\mathbf{V W}$-colorless). Onto the needle crystals, $\mathrm{F}_{4}-\mathrm{TCNQ}(2 \mathrm{~nm})^{8-9}$ and gold $(70 \mathrm{~nm})$ as contacts were deposited through a shadow mask to construct contacts and a bottom-gate-top-contact transistor. Finally, to determine channel size, unnecessary crystals were removed by laser etching technique.

The transfer characteristics for a representative FET with $\mathbf{C}_{\mathbf{1 0}}$-DNS-VW-colorless needle crystals were shown in Figure S30. The needle crystals work as p-type organic semiconductors, but they show significant hysteresis against $V_{\mathrm{G}}$ sweep and large threshold voltage, which imply that the 1D-needle crystals are not suitable for carrier transport. Furthermore, the transistor performance was affected by bias stress and output characteristics could not be successfully collected. Actually, their mobility is lower than $\mathbf{C}_{\mathbf{1 0}}$-DNS-VW-yellow by $4-5$ orders of magnitude (in order of $10^{-4} \mathrm{~cm}^{2} / \mathrm{Vs}$ ). The trend of carrier transport property agrees with calculation results.
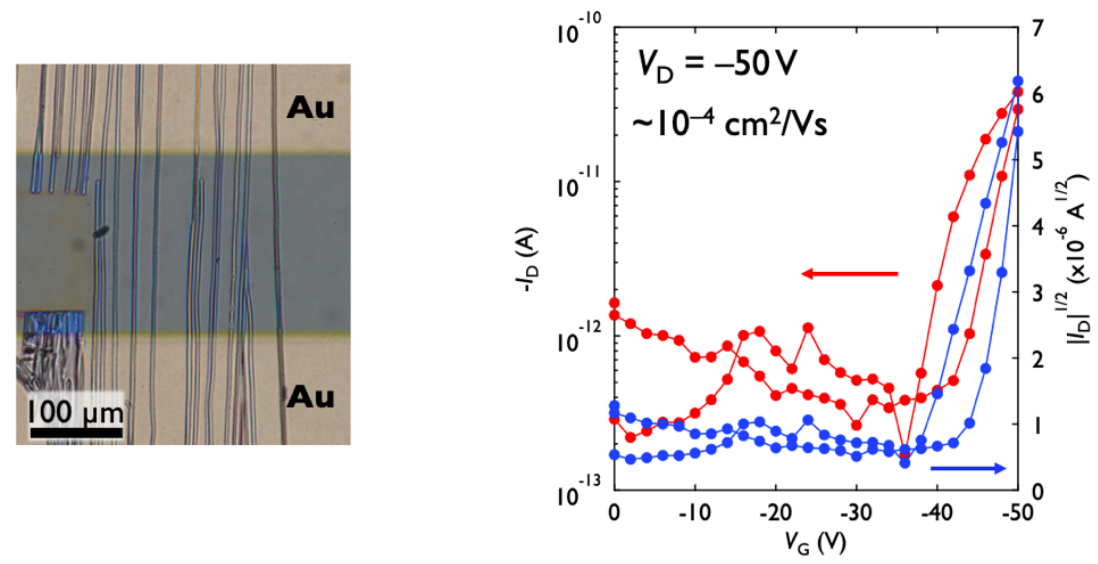

Figure S30. (left) Top view and (right) transfer characteristics of a $\mathbf{C}_{\mathbf{1 0}}-\mathbf{D N S}-\mathbf{V W}$-colorless single crystal transistor. 


\section{References}

(1) Kondo, Y.; Shilai, M.; Uchiyama, M.; Sakamoto, T. TMP-Zincate as Highly Chemoselective Base for Directed Ortho Metalation. J. Am. Chem. Soc. 1999, 121, 3539-3540.

(2) Uchiyama, M.; Miyoshi, T.; Kajihara, Y.; Sakamoto, T.; Otani, Y.; Ohwada, T.; Kondo, Y. Generation of Functionalized Asymmetric Benzynes with TMP-Zincates. Effects of Ligands on Selectivity and Reactivity of Zincates. J. Am. Chem. Soc. 2002, 124, 8514-8515.

(3) Uchiyama, M.; Kobayashi, Y.; Furuyama, T.; Nakamura, S.; Kajihara, Y.; Miyoshi, T.; Sakamoto, T.; Kondo, Y.; Morokuma, K. Generation and Suppression of 3-/4-Functionalized Benzynes Using Zinc Ate Base (TMP-Zn-ate): New Approaches to Multisubstituted Benzenes. J. Am. Chem. Soc. 2008, 130, 472-480.

(4) Maaninen, A.; Chivers, T.; Parvez, M.; Pietikäinen, J.; Laitinen, R. S. Syntheses of THF Solutions of SeX2 $(\mathrm{X}=\mathrm{Cl}, \mathrm{Br})$ and a New Route to Selenium Sulfides SenS8-n (n = 1-5): X-ray Crystal Structures of SeCl2(tht)2 and SeCl2·tmtu. Inorg. Chem. 1999, 38, 4093-4097.

(5) Kato, K.; Hirose, R.; Takemoto, M.; Ha, S.; Kim, J.; Higuchi, M.; Matsuda, R.; Kitagawa, S.; Takata, M. The RIKEN Materials Science Beamline at SPring-8: Towards Visualization of Electrostatic Interaction. AIP Conference Proceedings 2010, 1234, 875-878.

(6) Schmidt, M. W.; Baldridge, K. K.; Boatz, J. A.; Elbert, S. T.; Gordon, M. S.; Jensen, J. H.; Koseki, S.; Matsunaga, N.; Nguyen, K. A.; Su, S.; Windus, T. L.; Dupuis, M.; Montgomery Jr, J. A. General atomic and molecular electronic structure system. J. Comput. Chem. 1993, 14, 1347-1363.

(7) Valeev, E. F.; Coropceanu, V.; da Silva Filho, D. A.; Salman, S.; Brédas, J.-L. Effect of Electronic Polarization on Charge-Transport Parameters in Molecular Organic Semiconductors. J. Am. Chem. Soc. 2006, 128, 9882-9886.

(8) Minari, T.; Miyadera, T.; Tsukagoshi, K.; Aoyagi, Y.; Ito, H. Charge injection process in organic field-effect transistors. Appl. Phys. Lett. 2007, 91, 053508.

(9) Mitsui, C.; Okamoto, T.; Yamagishi, M.; Tsurumi, J.; Yoshimoto, K.; Nakahara, K.; Soeda, J.; Hirose, Y.; Sato, H.; Yamano, A.; Uemura, T.; Takeya, J. High-Performance Solution-Processable N-Shaped Organic Semiconducting Materials with Stabilized Crystal Phase. Adv. Mater. 2014, 26, 4546-4551. 